\title{
TOPOLOGY OF REAL CUBIC FOURFOLDS
}

\author{
S. Finashin, V. Kharlamov
}

\begin{abstract}
A solution of the problem of topological classification of real cubic fourfolds is given. It is proven that the real locus of a real non-singular cubic fourfold is diffeomorphic either to a connected sum $\mathbb{R P} P^{4} \# i\left(S^{2} \times S^{2}\right) \# j\left(S^{1} \times S^{3}\right)$, or to a disjoint union $\mathbb{R P} P^{4} \sqcup S^{4}$.
\end{abstract}

La voie la plus courte et la meilleure entre deux vérités de domaine réel passe souvent par le domaine imaginaire.

J.S. Hadamard, Essai sur la psychologie de l'invention dans le domaine mathématique, Edition Gaithier-Villars, 1975 , p. 114

\section{INTRODUCTION}

1.1. The subject. Studying the topology of cubic hypersurfaces is a classical task in real algebraic geometry. It has a long history going back to Newton, who undertook a systematic study of real plane cubics (first published in 1704 as an appendix to his book Opticks), and to Schläfli, Cayley, and Klein, who in a series of treatises (dating from 1858 to 1873 and certainly strongly motivated by Cayley-Salmon discovery of 27 straight lines on nonsingular cubic surfaces) classified the shapes of real cubic surfaces. Cubic surfaces (as well as cubic curves and higher dimensional cubic hypersurfaces) can be classified in different manners. As far as we know, it was Klein who first clearly addressed the problem of deformation and topological classifications. He solved it for nonsingular cubic surfaces and specially emphasized that for nonsingular cubic surfaces the deformation classification coincides with the topological one: two real nonsingular cubic surfaces in $P^{3}$ are deformation equivalent if and only if their real point sets are homeomorphic. Namely, there are 5 deformation classes of nonsingular cubic surfaces respectively to 5 topological types: $\mathbb{R} P^{2} \sqcup S^{2}, \mathbb{R P}^{2}, \#_{3} \mathbb{R} \mathrm{P}^{2}, \#_{5} \mathbb{R} \mathrm{P}^{2}$, and $\#_{7} \mathbb{R} \mathrm{P}^{2}$ (\# stands for the connected sum and $\sqcup$, for the disjoint sum). Recall, that the situation with the real plane cubics is similar: there are 2 deformation classes of nonsingular cubic curves and they are distinguished by their topological types, $S^{1} \sqcup S^{1}$ and $S^{1}$ (or one may like to write more instructively, $\mathbb{R P}^{1} \sqcup S^{1}$ and $\mathbb{R P}^{1}$ ).

Only recently a deformation classification of real cubic threefolds was completed by V. Krasnov [Kr1] who proved that there are 9 classes of real nonsingular cubic threefolds $X \subset P^{4}$. It turned out that these 9 classes of cubics $X$ are distinguished by the Betti numbers of the real point set $X(\mathbb{R})$ plus vanishing or non-vanishing of the homology

1991 Mathematics Subject Classification. 14P25, 14J10, 14N25, 14J35, 14J70.

Key words and phrases. Real Cubic Hypersurface, Fourfold, Periods, Deformation, Cuspidal Cubics. 
class realized by $X(\mathbb{R})$ in the middle homology group $H_{3}(X ; \mathbb{Z} / 2)$ of the complex point set (we denote the complex point set by the same letter as the variety itself). In a subsequent paper $\mathrm{Kr} 2$ Krasnov determined the topological type of $X(\mathbb{R})$ for 8 of the 9 deformation classes: for 1 class it is $\mathbb{R} P^{3} \sqcup S^{3}$ and for 7 others it is $\mathbb{R P}^{3} \#_{k}\left(S^{1} \times S^{2}\right)$, $k=0, \ldots, 6$.

In [FK1, FK2] we have undertaken a systematic study of deformations classes of real cubic fourfolds. At the first step, in [FK1] we obtained a coarse deformation classification (that is a classification up to deformations combined with the projective equivalence) of real cubic fourfolds. There we did not only enumerate the deformation classes (their number is 75), but also described them in terms of simple homological invariants, and, in addition, provided the adjacency graph (which we call the K4-graph) of the deformation classes. This graph essentially coincides with the adjacency graph for non-polarized real K3-surfaces (the K3-graph), which was a somewhat unexpected outcome of our research, even though cubic fourfolds are well-known to be related to the K3 surfaces in many ways.

The difference between the coarse and ordinary deformation classifications is encoded in the chirality phenomena: we say that a real nonsingular cubic $X \subset P^{5}$ and its coarse deformation class are chiral if $X$ and its image under a mirror reflection, $X^{\prime}$, belong to different connected components of the space of real nonsingular cubics. Thus, each chiral coarse deformation class gives two ordinary deformation classes, and each achiral coarse deformation class gives only one ordinary deformation class. In [FK2] we analyzed the chirality of the deformation classes of real nonsingular cubic fourfolds. We reduced the chirality problem to a specific problem of the arithmetics of lattices and used this reduction to show that certain real cubic fourfolds are chiral, while certain other real cubic fourfolds are achiral. The crucial role in the reduction to the arithmetics of lattices is played by the period map and the corresponding surjectivity statement (which was established in the complex setting by R. Laza $[\mathrm{La}$ and E. Looijenga $[\mathrm{Ld}$ shortly before our work).

1.2. The main result. Despite such a detailed understanding of the deformation classes, the topology of the real locus for real nonsingular cubic fourfolds being too much beyond the control under indicated above approaches remained far from being understood. The topological classification required additional tools.

In the present paper we introduce two such ingredients. One of them is based on detecting cuspidal cubics on the boundary of deformation components and analyzing the surgeries provided by perturbations of a cuspidal cubic. Another essential ingredient is a technique of ramified connected sums. Combining these tools with the results and certain methods from [FK1, FK2], we solve the problem of topological classification of real cubic fourfolds. Our main result is the following (more detailed statements are given in Section 5).

1.2.1. Theorem. The real locus of a real non-singular cubic fourfold is diffeomorphic either to a connected sum $\mathbb{R} \mathrm{P}^{4} \# i\left(S^{2} \times S^{2}\right) \# j\left(S^{1} \times S^{3}\right)$ (the connected case), or to a disjoint union of $\mathbb{R P}^{4}$ with $S^{4}$ (disconnected case).

In addition, we complete the topological description of real nonsingular cubic threefolds, $X \subset P^{4}$, by applying our technique to the deformation class which was not analyzed by Krasnov. In this case the real locus, $X(\mathbb{R})$, turns out to be a Seifert manifold described in Section 6 (Theorem 6.1.1).

1.3. Structure of the paper. The paper is organized as follows. 
In Section 2 we review some basic facts about real cubic fourfolds. In particular, in Subsection 2.2 we describe the K4-graph, whose vertices $C^{i, j}$ and $C_{I}^{i, j}$ represent the deformation classes of real cubic fourfolds and whose edges describe adjacency of these classes. The first step towards proving Theorem 1.2.1 is made in Subsection 2.3, where using elementary arguments, we deduce that vertices $C^{0,0}$ and $C_{I}^{1,0}$ represent cubic fourfolds $X$ whose real locus $X(\mathbb{R})$ is $\mathbb{R} P^{4}$ and $\mathbb{R} \mathrm{P}^{4} \sqcup S^{4}$ respectively. Subsections 2.6 - 2.7 contain basic definitions and facts related to the period map for cubic fourfolds. This techniques (which played a crucial role in [FK2]) is involved here only in one place: in the proof of Lemma 3.3.2 (this Lemma provides an arithmetical criterion of existence of cuspidal cubics on the boundary of a given deformation component).

In Section 3 we analyze the Morse modifications experienced by $X(\mathbb{R})$ as we move down the K4-graph. This technique allows us to use some kind of inductive scheme for proving Theorem 1.2.1 with $C^{0,0}$ as the basis of induction. However it turns out that in two cases, $C_{I}^{2,1}$ and $C^{10,1}$, such Morse theory inductive arguments do not work, and we need to apply a more involved technique. Such a technique of "ramified connected sums" is developed in Section 1 .

In Section 5 we apply the results and the technique of the previous sections to prove Theorem 1.2.1. In Section 6 we make some concluding remarks. In particular, we apply the technique of ramified connected sums to complete the topological classification of real cubic threefolds (Theorem 6.1.1). We discuss also certain perspectives in studying higher dimensional cubics.

1.4. Conventions. When in a subsection all or most of the material is a recollection of already known results, the appropriate references are given in brackets after the subsection title (some results may be not explicitly mentioned in those cited papers, in such a case we provide their proof). As usual, symbol ' $\square$ ' after a statement means that no proof will follow: either the proof is straightforward, or it has already been explained, or a reference is given (in the statement, or at the beginning of the subsection).

Notation $X(\mathbb{R})$ refers always to the real locus of a variety $X$ defined over $\mathbb{R}$. However we write $\mathbb{R P}^{n}$ instead of $P^{n}(\mathbb{R})$ in the topological context. In Section 4 , we also diverge from algebro-geometric notation style by using single letters $(P, Q, V, L)$ for certain real loci as soon as they are involved in topological constructions.

1.5. Acknowledgements. The principal ideas of this paper were developed by the authors during their visit to Centre Interfacultaire Bernoulli in EPFL (Lausanne). The text was finalized during the first author's visit to Universite de Strasbourg. The last touch was made during RIP-stay in Mathematiches Forschungsinstitut Oberwolfach. We thank these institutions for hospitality.

\section{Deformation CHAMBERS AND THE PERIOD MAP}

2.1. Discriminant hypersurface and deformation chambers ( FK1]). The complex projective cubic fourfolds form the complex projective space $P_{4,3}=P\left(\operatorname{Sym}^{3}\left(\mathbb{C}^{6}\right)^{*}\right)$ of dimension $\left(\begin{array}{c}5+3 \\ 3\end{array}\right)-1=55$ and the singular ones form in it the, so called, discriminant hypersurface $\Delta \subset P_{4,3}$. The real cubic fourfolds, that is cubic fourfolds defined by real polynomials, form the real projective space $P_{4,3}(\mathbb{R})$, so that the real singular cubic fourfolds form the real discriminant hypersurface $\Delta(\mathbb{R}) \subset P_{4,3}(\mathbb{R})$.

We study the non-singular real cubics, so the space of our interest is nothing but the complement $\mathscr{C}=P_{4,3}(\mathbb{R}) \backslash \Delta(\mathbb{R})$ of the discriminant hypersurface. We say that nonsingular real cubic fourfolds $X_{1}$ and $X_{2}$ are deformation equivalent if they belong to the 
same component of $\mathscr{C}$. The connected components of $\mathscr{C}$ will be called the deformation components, or the deformation chambers.

Remark. In our definition we consider a real cubic as a singular one, even if it has only imaginary singular points. Thus, it may be worth mentioning that the real cubics with real singular points form a real semi-algebraic subset $\Delta^{\prime} \subset \Delta$, such that the difference $\Delta \backslash \Delta^{\prime}$ has codimension 2 in $P_{4,3}(\mathbb{R})$. Because of this phenomenon the imbedding of $P_{4,3}(\mathbb{R}) \backslash \Delta$ into $P_{4,3}(\mathbb{R}) \backslash \Delta^{\prime}$ induces a bijection at the level of connected components and the two corresponding deformation classifications coincide.

The smooth part $\Delta_{1}$ of $\Delta$ is formed by cubics with a node ( $A_{1}$-singularity) and no other singular points. The connected components of $\Delta_{1}(\mathbb{R})$ are called facets. Two facets can be adjacent through a stratum formed by cubics with a cusp $\left(A_{2}\right.$-singularity), in such a case the two facets are called cuspidally adjacent. The union of the facets and the strata formed by cubics with a cusp is a topological codimension-one submanifold of $P_{4,3}(\mathbb{R})$. We denote this union by $\Delta_{2}(\mathbb{R})$ and call its connected components the walls. If two deformation components $C$ and $C^{\prime}$ have a common wall, we say that they are adjacent.

A cubic $X \in \mathscr{C}$ is called achiral if it is deformation equivalent to its image $\rho(X)$ under the mirror reflection $\rho: P^{5}(\mathbb{R}) \rightarrow P^{5}(\mathbb{R})$ against some hyperplane in $P^{5}(\mathbb{R})$; otherwise the cubic $X$ is called chiral.

The relation of coarse deformation equivalence is a combination of deformation and projective equivalences. Namely, cubics $X_{1}$ and $X_{2}$ are coarse deformation equivalent if $X_{1}$ is deformation equivalent to the image of $X_{2}$ under a projective transformation (that is either to $X_{2}$ or to $\rho\left(X_{2}\right)$ ).

In accord with this, by the coarse deformation component we mean a connected component of the quotient-space $\widetilde{\mathscr{C}}=\mathscr{C} / P G L(6, \mathbb{R})$ with respect to the action of the projective group $P G L(6, \mathbb{R})$ in $P_{4,3}(\mathbb{R})$ induced by the action in $P^{5}(\mathbb{R})$.

It is obvious that one coarse deformation component of chiral cubics corresponds to two ordinary deformation components, and that for achiral cubics the deformation and the coarse deformation components are in one-to-one correspondence.

A pair of coarse deformation components are called adjacent if there is a pair of adjacent deformation components representing these coarse components.

2.2. K4- and K3-graphs( cubic fourfolds as vertices and their adjacency as edges, we obtain a graph. We call it the K4-graph and denote it by $\Gamma_{K 4}$. This name is essentially to reflect its remarkable similarity to the better known K3-graph which describes similarly the set of deformation components of real non-polarized K3-surfaces and their adjacency.

2.2.1. Theorem. The graphs $\Gamma_{K 4}$ and $\Gamma_{K 3}$ are the graphs shown respectively on Figures 1 and 2.

The coordinates $r$ and $d$ on the Figures characterize the action of the complex conjugation in the corresponding lattices. Namely, in the case of a real cubic fourfold $X$, we consider the involution $c_{X}: \mathbb{M} \rightarrow \mathbb{M}$ induced in $\mathbb{M}=H^{4}(X)$ by the complex conjugation, and denote by $d$ the rank of the 2-periodic discriminant group $\mathbb{M} /\left(\mathbb{M}_{+}+\mathbb{M}_{-}\right)=\mathbb{M}_{ \pm}^{*} / \mathbb{M}_{ \pm}$, where $\mathbb{M}_{ \pm}=\left\{x \in \mathbb{M} \mid c_{X}(x)= \pm x\right\}$. By well known Smith theory arguments, $d=\frac{1}{2}\left(b_{*}(X)-b_{*}(X(\mathbb{R}))\right)=\frac{1}{2}\left(27-b_{*}(X(\mathbb{R}))\right)$ where $b_{*}=\sum_{i \geqslant 0} b_{i}$ is the total Betti number with $\mathbb{Z} / 2$-coefficients. In the case of a real $\mathrm{K} 3$-surface $Y$, there is a similar involution $c_{Y}: \mathbb{L} \rightarrow \mathbb{L}$ in $\mathbb{L}=H^{2}(Y)$ and $d$ denotes the rank of the discriminant group $\mathbb{L} /\left(\mathbb{L}_{+}+\mathbb{L}_{-}\right)=\mathbb{L}_{ \pm}^{*} / \mathbb{L}_{ \pm}$, where $\mathbb{L}_{ \pm}=\left\{x \in \mathbb{L} \mid c_{Y}(x)= \pm x\right\}$. If 
Figure 1. The K4-GRAPH $\Gamma_{K 4}$

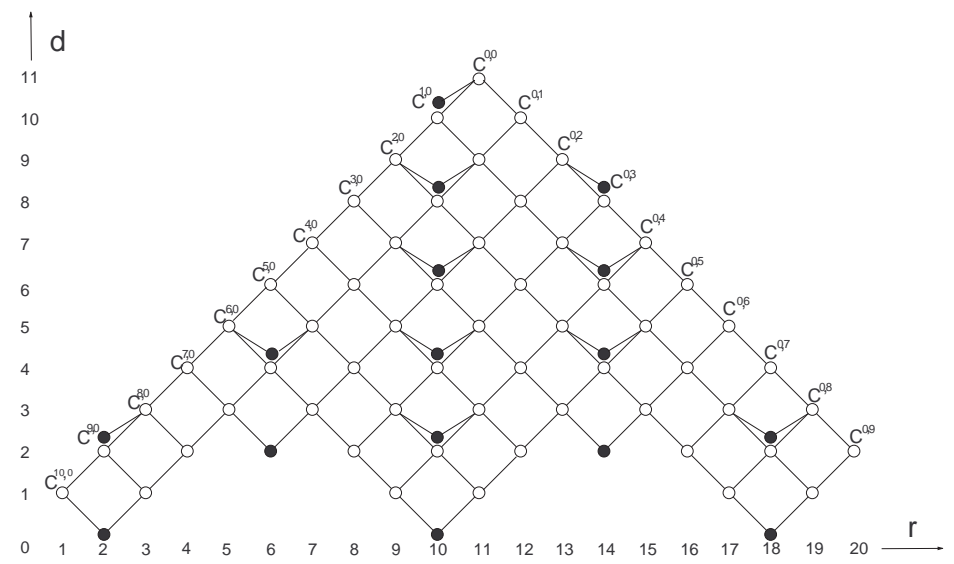

Figure 2. The K3-GRAPH $\Gamma_{K 3}$

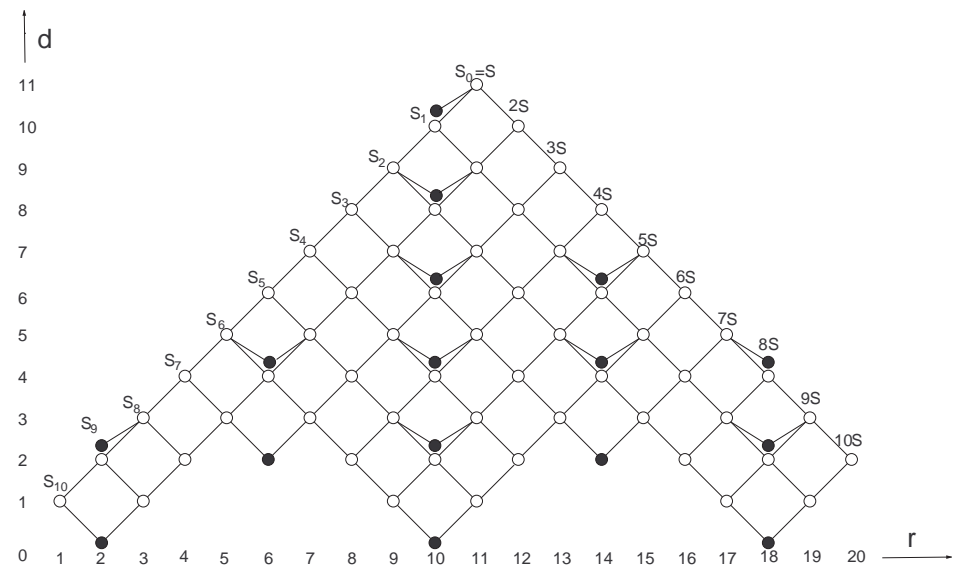

$Y(\mathbb{R}) \neq \varnothing$, then we have similarly $d=\frac{1}{2}\left(b_{*}(Y)-b_{*}(Y(\mathbb{R}))\right)=\frac{1}{2}\left(22-b_{*}(Y(\mathbb{R}))\right)$, and in the exceptional case $Y(\mathbb{R})=\varnothing$, we have $d=10$.

The coordinate $r$ is the rank of $\mathbb{M}_{-}$in the case of cubic fourfolds and the rank of $\mathbb{L}_{+}$ in the case of K3-surfaces. Respectively, as it follows from the Lefschetz trace formula, $r$ is equal to $\frac{1}{2}(\chi(X)-\chi(X(\mathbb{R}))-4)=11+\frac{1}{2}(1-\chi(X(\mathbb{R})))$ in the first case, and $\frac{1}{2}(\chi(Y)+\chi(Y(\mathbb{R}))-4)=10+\frac{1}{2} \chi(Y(\mathbb{R}))$ in the second.

In $\Gamma_{K 4}$ as well as in $\Gamma_{K 3}$ there are 10 pairs of vertices-twins having the same coordinates $(r, d)$, and on Figures 1 and 2 we place one of the vertices in each pair slightly above the other vertex (one may consider this as introducing a third coordinate).

The vertices in $\Gamma_{K 4}$ and in $\Gamma_{K 3}$ which are marked by $\bullet$ have lattices of the type I. The other vertices are marked by $\circ$ and have lattices of the type II (we recall the definition of the types in 2.4). Note that in a pair of vertices-twins with the same coordinates $(r, d)$ one vertex belongs to the type I and the other to type II. Among the vertices which are completely determined by $(r, d)$ the majority belong to the type II, but the five bottom "local minima" vertices belong to the type I.

It will be convenient for us to introduce, instead of $(r, d)$, a new coordinate system 
$(i, j)$ centered at the top vertex, namely, the system where $i=\frac{1}{2}(22-r-d)$ is the number of down-left and $j=\frac{1}{2}(r-d)$ of down-right moves along edges needed to reach a given vertex from the top. We will call such down-left moves $L$-moves and the downright moves $R$-moves. The moves in the opposite directions will be called respectively $L^{-1}$-moves and $R^{-1}$-moves. A vertex with coordinates $(i, j)$ will be denoted $C^{i, j}$ (in the case of the K3-graph as well as for the K4-graph) if it is the only vertex with such coordinates. In the case of two vertices with coordinates $(i, j)$ we use notation $C_{I}^{i, j}$ for the vertex of type I and $C^{i, j}$ for the other vertex. The set formed by the vertices $C^{i, j}$ will be called the principal series of vertices, and the vertices $C_{I}^{i, j}$ will be called the special vertices. We say that a vertex of the K3-graph and a vertex of the K4-graph correspond to each other if they are of the same type and have the same $(i, j)$ coordinates (i.e., the both have the same notation, either $C^{i, j}$ or $C_{I}^{i, j}$ ).

The walls (the facets) between the deformation chambers are divided into $L$-walls and $R$-walls ( $L$-facets and $R$-facets), depending on the edges they represent in $\Gamma_{K 4}$.

2.3. Immediate topological consequences of deformation classification. It is easy to construct in any dimension $n$ a non-singular real cubic hypersurface $X \subset P^{n+1}$ whose real locus $X(\mathbb{R})$ is diffeomorphic to $\mathbb{R} \mathrm{P}^{n}$, as well as a cubic with $X(\mathbb{R})=\mathbb{R} \mathrm{P}^{n} \sqcup S^{n}$. Namely, it is sufficient to perturb a singular cubic which splits into a hyperplane and a quadric; if the quadric has an empty real locus, then we obtain $X(\mathbb{R})=\mathbb{R} \mathrm{P}^{n}$, and if it has a real locus disjoint from the hyperplane, then $X(\mathbb{R})=\mathbb{R P}^{n} \sqcup S^{n}$. Clearly, in each of these two cases the models constructed in such a way are deformation equivalent to each other.

2.3.1. Lemma. If the real locus $X(\mathbb{R})$ of a non-singular real cubic $n$-fold $X, n \geqslant 1$, is disconnected then $X(\mathbb{R})$ is diffeomorphic to a disjoint union of $\mathbb{R P}^{n}$ and $S^{n}$. Such cubics form a single deformation component.

Proof. As it follows from Bézout theorem, $X(\mathbb{R})$ realizes a non-trivial class in the homology group $H_{n}\left(\mathbb{R P}^{n+1} ; \mathbb{Z} / 2\right)$. So does also one of its connected components, $C_{1}$. Any other connected component, $C_{2}$, should realize a trivial class since $C_{1} \cap C_{2}=\varnothing$. The complement $\mathbb{R P}^{n+1} \backslash C_{2}$ splits in two connected components one of which contains $C_{1}$, and we choose a point $p$ in the other component. Any real line through $p$ intersects $C_{1}$ at least once and $C_{2}$ at least twice. So (again by Bézout theorem) $X(\mathbb{R})$ cannot have other components and the central projection $X(\mathbb{R}) \rightarrow \mathbb{R P}^{n}$ from $p$ gives a 1-1 map $C_{1} \rightarrow \mathbb{R P}^{n}$ and a double covering $C_{2} \rightarrow \mathbb{R P}^{n}$. This proves the first statement of the Lemma.

The same arguments show that if a real cubic $(n-1)$ )-fold (in a real projective $n$ space) is disconnected and has a real singular point, then its real locus is the union of this point and a real projective hyperplane disjoint from it. Therefore, at any point of $C_{2}$ the hyperplane tangent to $C_{2}$ intersects $C_{2}$ exclusively by this tangency point. Let us shift the tangency hyperplane to make it a real hyperplane $H$ disjoint from $C_{2}$, pick up a small ellipsoid $E$ inside $C_{2}$ and consider the cubic $X^{\prime}$ which is a small real perturbation of $H \cup E$. Such a cubic $X^{\prime}$ is deformation equivalent to $X$, since they can be joined by a straight deformation path, $t f+(1-t) g$, where the degree 3 defining equations $f=0$ and $g=0$ of $X$ and $X^{\prime}$, respectively, are chosen in such a way that $f g>0$ at the solid ellipsoid bounded by $E$ in $P^{n+1}(\mathbb{R})$ : under this sign convention the cubics $t f+(1-t) g=0, t \in[0,1]$, remain disconnected and, hence, non-singular. Now, the second statement of the Lemma follows from the mutual deformation equivalence of all the model disconnected cubics. 
2.3.2. Corollary. (a) If a non-singular real cubic fourfold has real part $X(\mathbb{R})=\mathbb{R} \mathrm{P}^{4}$, then it represents the vertex $C^{0,0}$. (b) If a non-singular real cubic fourfold has real part $X(\mathbb{R})=\mathbb{R} \mathrm{P}^{4} \cup S^{4}$, then it represents the vertex $C_{I}^{1,0}$.

Proof. In the case (a), the relations $r=11+\frac{1}{2}(1-\chi(X(\mathbb{R})))=11, d=\frac{1}{2}(27-$ $\left.b_{*}(X(\mathbb{R}))\right)=11$ give $i=\frac{1}{2}(22-11-11)=0, j=\frac{1}{2}(11-11)=0$, and we see from the graph $\Gamma_{K 4}$ that $C^{0,0}$ is the unique suitable vertex. Similarly, in the case (b) we obtain $i=1, j=0$ and then notice in addition that the only connected four-manifold that can be obtained from $\mathbb{R P}^{4} \cup S^{4}$ by a single Morse modification is $\mathbb{R} \mathrm{P}^{4}$, while as is seen on the graph $\Gamma_{K 4}$ the vertex $C^{1,0}$ is adjacent to two vertices. Thus, the corresponding vertex is $C_{I}^{1,0}$.

2.4. Markings and eigenlattices ([FK1,FK2]). By the lattice $\mathbb{M}(X)$ of a nonsingular cubic fourfold $X$ we mean the middle cohomology group $H^{4}(X ; \mathbb{Z})$ endowed with the intersection form. This is an odd unimodular lattice of signature $(21,2)$. The polarization class $h_{X} \in \mathbb{M}(X)$ (which is realized by the intersection of $X$ with a projective 3 -space) has square $h_{X}^{2}=3$ and is characteristic which means as usual that $x h_{X}=x^{2} \bmod 2$ for all $x \in \mathbb{M}(X)$. This implies that there exists a lattice isomorphism between $\mathbb{M}(X)$ and $\mathbb{M}=3 I+2 U+2 E_{8}$ such that $h_{X}$ is mapped into $h=(1,1,1) \in 3 I$; in particular, the primitive sublattice $\mathbb{M}^{0}=\{x \in \mathbb{M} \mid x h=0\}$ becomes identified with $A_{2}+2 U+2 E_{8}$. Such a choice of a lattice isomorphism $\phi: \mathbb{M}(X) \rightarrow \mathbb{M}$ will be called $a$ marking of $X$, and a pair $(X, \phi)$ will be called a marked cubic fourfold.

If a cubic $X$ is defined over reals, then the complex conjugation induces a lattice involution $c_{X}: \mathbb{M}(X) \rightarrow \mathbb{M}(X)$ such that $c_{X}\left(h_{X}\right)=h_{X}$. This involution gives rise to the eigenlattices $M_{ \pm}(X)=\left\{x \in \mathbb{M}(X) \mid c_{X}(x)= \pm x\right\}$ and $\mathbb{M}_{ \pm}^{0}(X)=\left\{x \in \mathbb{M}^{0}(X) \mid c_{X}(x)=\right.$ $\pm x\}$. Here, $\mathbb{M}_{-}^{0}(X)=\mathbb{M}_{-}(X)$ since $h_{X} \in \mathbb{M}_{+}(X)$. A marking $\phi: \mathbb{M}(X) \rightarrow \mathbb{M}$ transforms the involution $c_{X}$ and its eigenlattices $\mathbb{M}_{ \pm}(X), \mathbb{M}_{ \pm}^{0}(X)$ into an involution on $\mathbb{M}$ and the eigenlattices of this induced involution.

An involution $c: \mathbb{M} \rightarrow \mathbb{M}$ is induced by $c_{X}$ for some marked real cubic fourfold $(X, \phi)$ if and only if the involution $c$ is geometric, where the latter means that $c(h)=h$ and each of the eigenlattices $\mathbb{M}_{ \pm}=\{x \in \mathbb{M} \mid c(x)= \pm x\}$ and $\mathbb{M}_{ \pm}^{0}=\{x \in \mathbb{M} \mid c(x)= \pm x, x h=0\}$ is of negative inertia index equal to 1 . In fact, the isomorphism class of the pair $\left(\mathbb{M}(X), c_{X}\right)$ is an invariant which allows to distinguish the coarse deformation classes of real cubic fourfolds $X$. Moreover, the isomorphism class of $\left(\mathbb{M}(X), c_{X}\right)$ is determined by the isomorphism class of any of the eigenlattices $\mathbb{M}_{+}^{0}(X)$ and $\mathbb{M}_{-}(X)$.

The isomorphism classes of lattices $\mathbb{M}_{+}^{0}$ and $\mathbb{M}_{-}$corresponding to geometric involutions are listed in the following tables $1-3$.

We say that a real cubic $X$ is of type $\mathrm{I}$ if the involution $c_{X}: \mathbb{M}(X) \rightarrow \mathbb{M}(X)$ is even, that is if $x \cdot c_{X}(x)+x^{2}=0 \bmod 2 \mathbb{Z}$ for any $x \in \mathbb{M}(X)$. As is known, it happens if and only if $X(\mathbb{R})$ realizes the class $h_{X}$ in the middle $(\mathbb{Z} / 2)$-homology of $X$.

The above definition of type $I$ is not specific to cubics, but is applied to any nonsingular real algebraic variety. In particular, if $Y$ is a $K 3$-surface, it is of type $I$ if and only if $Y(\mathbb{R})$ realizes zero in the middle $(\mathbb{Z} / 2)$-homology of $Y$.

2.5. Central projection K3-K4 correspondence [FK1]. The edges of the K4graph can be interpreted as coarse deformation classes of 6-polarized K3-surfaces via the following central projection correspondence. Let $X_{0}$ denote a nodal real cubic fourfold representing an edge. In an affine chart centered at the node, the cubic $X_{0}$ is defined as $\left\{f_{2}+f_{3}=0\right\} \subset \mathbb{R}^{5}$ where $f_{2}$ and $f_{3}$ are some quadratic and, respectively, cubic homogeneous polynomials in $x_{0}, \ldots, x_{4}$. Non-degeneracy of the node means that the 
Table 1.

$\mathbb{M}_{+}^{0}$ for cubic fourfolds of type $C^{i, j}$

\begin{tabular}{|lc|}
\hline$C^{0, j}$ & $-A_{1}+(9-j) A_{1}+\langle 6\rangle$ \\
$C^{1, j}$ & $-A_{1}+(9-j) A_{1}+A_{2}$ \\
$C^{2, j}$ & $U+(9-j) A_{1}+A_{2}$ \\
$C^{3, j}$ & $U+(6-j) A_{1}+A_{2}+D_{4}$ \\
$C^{4, j}$ & $-A_{1}+(5-j) A_{1}+\langle 6\rangle+E_{8}$ \\
$C^{5, j}$ & $-A_{1}+(5-j) A_{1}+A_{2}+E_{8}$ \\
$C^{6, j}$ & $U+(5-j) A_{1}+A_{2}+E_{8}$ \\
$C^{7, j}$ & $U+(2-j) A_{1}+A_{2}+D_{4}+E_{8}$ \\
$C^{8, j}$ & $-A_{1}+(1-j) A_{1}+\langle 6\rangle+2 E_{8}$ \\
$C^{9, j}$ & $-A_{1}+(1-j) A_{1}+A_{2}+2 E_{8}$ \\
$C^{10, j}$ & $U+(1-j) A_{1}+A_{2}+2 E_{8}$ \\
\hline
\end{tabular}

Table 2.

$\mathbb{M}_{-}$for cubic fourfolds of type $C^{i, j}$

\begin{tabular}{|lc|}
\hline$C^{i, 0}$ & $-A_{1}+(10-i) A_{1}$ \\
$C^{i, 1}$ & $U+(10-i) A_{1}$ \\
$C^{i, 2}$ & $U+(7-i) A_{1}+D_{4}$ \\
$C^{i, 3}$ & $-A_{1}+(6-i) A_{1}+E_{7}$ \\
$C^{i, 4}$ & $-A_{1}+(6-i) A_{1}+E_{8}$ \\
$C^{i, 5}$ & $U+(6-i) A_{1}+E_{8}$ \\
$C^{i, 6}$ & $U+(3-i) A_{1}+D_{4}+E_{8}$ \\
$C^{i, 7}$ & $-A_{1}+(2-i) A_{1}+E_{7}+E_{8}$ \\
$C^{i, 8}$ & $-A_{1}+(2-i) A_{1}+2 E_{8}$ \\
$C^{i, 9}$ & $U+(2-i) A_{1}+2 E_{8}$ \\
\hline
\end{tabular}

Table 3 .

\begin{tabular}{|lccc|}
\multicolumn{1}{l}{$\mathbb{M}_{+}^{0}$ and $\mathbb{M}_{-}$for cubic fourfolds of type $C_{I}^{i, j}$} \\
\hline$C_{I}^{i, j}$ & $\mathbb{M}_{+}^{0}$ & $\mathbb{M}_{-}$ \\
$C_{I}^{0,3}$ & $U(2)+E_{6}(2)$ & & $U(2)+3 D_{4}$ \\
$C_{I}^{1,8}$ & $U(2)+A_{2}$ & & $U(2)+2 E_{8}$ \\
$C_{I}^{1,4}$ & $U+E_{6}(2)$ & $U+3 D_{4}$ \\
$C_{I}^{2,5}$ & $U(2)+A_{2}+D_{4}$ & $U(2)+D_{4}+E_{8}$ \\
$C_{I}^{3,2}$ & $U(2)+A_{2}+2 D_{4}$ & $U(2)+2 D_{4}$ \\
$C_{I}^{4,3}$ & $U+A_{2}+2 D_{4}$ & $U+2 D_{4}$ \\
$C_{I}^{5,4}$ & $U(2)+A_{2}+E_{8}$ & $U(2)+E_{8}$ \\
$C_{I}^{6,1}$ & $U(2)+A_{2}+D_{4}+E_{8}$ & $U(2)+D_{4}$ \\
$C_{I}^{9,0}$ & $U(2)+A_{2}+2 E_{8}$ & $U(2)$ \\
$C_{I}^{2,1}$ & $U+A_{2}+E_{8}(2)$ & $U+E_{8}(2)$ \\
$C_{I}^{1,0}$ & $U(2)+A_{2}+E_{8}(2)$ & $U(2)+E_{8}(2)$ \\
\hline
\end{tabular}

quadric $\left\{f_{2}=0\right\} \subset \mathbb{R} \mathrm{P}^{4}$ is non-singular and absence of other singularities in $X_{0}$ is equivalent to transversality of the intersection $\left\{f_{2}=f_{3}=0\right\} \subset \mathbb{R} \mathrm{P}^{4}$, which is therefore a non-singular real 6-polarized K3-surface.

\subsubsection{Theorem.}

(1) A vertex $v_{K 3}$ of the K3-graph corresponds to a vertex $v_{K 4}$ of the K4-graph if and only if the eigenlattice $\mathbb{M}_{-}(X)$ of a cubic fourfold $X$ representing $v_{K 4}$ is isomorphic to the eigenlattice $-\mathbb{L}_{+}(Y)$ of a K3-surface $Y$ representing $v_{K 3}$.

(2) The edges of the K4-graph are in one-to-one correspondence with the coarse deformation classes of 6-polarized K3 surfaces $Y$. Namely, edges with the leftmost vertex $v_{K 4}$ correspond to the classes of $(Y, l)$, such that $Y$ represent $v_{K 3}$ and $l \in \mathbb{L}_{-}(Y), l^{2}=6$.

2.6. The period map ( $[\mathrm{La} \mid,[\mathrm{Lo}|,| \sqrt{\mathrm{VO}} \mid)$. The non-zero Hodge numbers in dimension four for any non-singular cubic fourfold $X \subset P^{5}$ are $h^{3,1}=h^{1,3}=1$ and $h^{2,2}=21$. Given a marking $\phi:\left(\mathbb{M}(X), h_{X}\right) \rightarrow(\mathbb{M}, h)$, the complex line $\phi\left(H^{3,1}(X)\right) \subset \mathbb{M}^{0} \otimes \mathbb{C}$ is isotropic and has negative pairing with the conjugate (and thus, also isotropic) line $\phi\left(H^{1,3}(X)\right)=\overline{\phi\left(H^{3,1}(X)\right)}$, that is to say, $w^{2}=0$, and $w \bar{w}<0$, (and thus $\bar{w}^{2}=0$ ) for all 
$w \in \phi\left(H^{3,1}(X)\right)$. Writing $w=u+i v, u, v \in \mathbb{M}^{0} \otimes \mathbb{R}$, we can reformulate it as $u^{2}=v^{2}<0$ and $u v=0$, which implies that the real plane $\langle u, v\rangle \subset \mathbb{M}^{0} \otimes \mathbb{R}$ spanned by $u$ and $v$ is negative definite and bears a natural orientation given by $u=\operatorname{Re} w, v=\operatorname{Im} w$. Note that the orientation determined similarly by the complex line $\phi\left(H^{1,3}(X)\right) \subset \mathbb{M}^{0} \otimes \mathbb{C}$ is the opposite one.

The line $\phi\left(H^{3,1}(X)\right) \subset \mathbb{M}^{0} \otimes \mathbb{C}$ specifies a point $\Omega(X) \in P\left(\mathbb{M}^{0} \otimes \mathbb{C}\right)$ (as usual, $P$ states for the projectivization) called the period point of $(X, \phi)$. This period point belongs to the quadric $\mathcal{Q}=\left\{w^{2}=0\right\} \subset P\left(\mathbb{M}^{0} \otimes \mathbb{C}\right)$, and more precisely, to its open subset, $\widehat{\mathscr{D}}=\{w \in \mathcal{Q} \mid w \bar{w}<0\}$. This subset has two connected components, which are exchanged by the complex conjugation (this reflects also switching from the given complex structure on $X$ to the complex conjugate one).

The orthogonal projection of a negative definite real plane in $\mathbb{M}^{0} \otimes \mathbb{R}$ to another such one is non-degenerate. Thus, to select one of the two connected components of $\widehat{\mathscr{D}}$ we fix an orientation of negative definite real planes in $\mathbb{M}^{0} \otimes \mathbb{R}$ which is constrained to be preserved by the orthogonal projection. We call it the prescribed orientation and restrict the choice of markings to those for which the orientation of $\phi\left(H^{3,1}(X)\right)$ defined by the pairs $u=\operatorname{Re} w, v=\operatorname{Im} w$ for $w \in \phi\left(H^{3,1}(X)\right)$ is the prescribed one. We denote this component by $\mathscr{D}$ and call it the period domain. By Aut ${ }^{+}\left(\mathbb{M}^{0}\right)$ we denote the group of those automorphisms of $\mathbb{M}^{0}$ which preserve the prescribed orientation (and thus preserve $\mathscr{D})$. We put $\operatorname{Aut}^{-}\left(\mathbb{M}^{0}\right)=\operatorname{Aut}\left(\mathbb{M}^{0}\right) \backslash \operatorname{Aut}{ }^{+}\left(\mathbb{M}^{0}\right)$. This complementary coset consists of automorphisms exchanging the connected components of $\widehat{\mathscr{D}}$.

On the other hand we have the projective space $P_{4,3}$ formed by all cubic fourfolds, which splits into the discriminant hypersurface $\Delta_{4,3}$ formed by singular cubics and its complement, $\mathscr{C}=P_{4,3} \backslash \Delta_{4,3}$. Let $\mathscr{C}^{\sharp}$ denote the space of marked non-singular cubics. The natural projection $\mathscr{C}^{\sharp} \rightarrow \mathscr{C}$ is obviously a Galois covering with the deck transformation group $\operatorname{Aut}^{+}\left(\mathbb{M}^{0}\right)$. The above conventions define the period map per: $\mathscr{C}^{\sharp} \rightarrow \mathscr{D}$, $(X, \phi) \mapsto \phi\left(H^{3,1}(X)\right)$.

The above definitions extend naturally to cubic fourfolds with simple singularities. In particular, the lattice $H^{4}(X)$ of a cubic fourfold with simple singularities is torsion free and admits an isometric embedding $H^{4}(X) \rightarrow \mathbb{M}$ whose orthogonal complement is isometric to $\bigoplus_{i} \mathbb{M}_{x_{i}}(X)$, the sum of the Milnor lattices $\mathbb{M}_{x_{i}}$ over all singular points $x_{i}$ of $X$. On the other hand, $H^{4}(X) \otimes \mathbb{C}$ carries a pure Hodge structure with $h^{3,1}=h^{1,3}=1$. By Riemann extension theorem and the finiteness of the monodromy groups of simple singularities, the Galois covering $\mathscr{C}^{\sharp} \rightarrow \mathscr{C}$ extends (in an unique way) to a ramified Galois covering $\mathscr{C}_{s}^{\sharp} \rightarrow \mathscr{C}_{s}$ where $\mathscr{C}_{s} \subset P_{4,3}$ is the space of cubic fourfolds with simple singularities. The covering space $\mathscr{C}_{s}^{\sharp}$ is non-singular. It is the space of marked cubic fourfolds with simple singularities, where by a marking of $X \in \mathscr{C}_{s}$ we understand, in accordance with the previous definitions, a respecting the prescribed orientation (of negative definite planes) isometric embedding $\left(H^{4}(X), h_{X}\right) \rightarrow(\mathbb{M}, h)$ whose orthogonal complement is isometric to the sum of the Milnor lattices over all singular points of $X$. The extended period map per: $\mathscr{C}_{s}^{\sharp} \rightarrow \mathscr{D},(X, \phi) \mapsto \phi\left(H^{3,1}(X)\right)$, is holomophic due to the corresponding Griffiths theorem.

Consider, on the period space side, the reflection $R_{v}$ in $\mathbb{M}^{0} \otimes \mathbb{C}$ across the mirrorhyperplane $H_{v}=\left\{x \in \mathbb{M}^{0} \otimes \mathbb{C} \mid x v=0\right\}$ defined as $x \mapsto x-2 \frac{x v}{v^{2}} v$, and note that it preserves the lattice $\mathbb{M}^{0}$ invariant if $v \in \mathbb{M}^{0}$ is such that $v^{2}=2$, or such that $v^{2}=6$ and $x v$ is divisible by 3 for all $x \in \mathbb{M}^{0}$. We call these two types of lattice elements 2-roots and 6-roots respectively, and denote their sets by $V_{2}$ and $V_{6}$. Note that $R_{v} \in \mathrm{Aut}^{+}\left(\mathbb{M}^{0}\right)$ for any $v \in V_{2} \cup V_{6}$. If $v \in V_{2}$, then the reflection $R_{v}$ extends (as a reflection) to $\mathbb{M}$ and $h$ 
is preserved by this extension. By contrary if $v \in V_{6}$, the reflection $R_{v}$ does not extend to a reflection in $\mathbb{M}$, and moreover, the unique extension of $R_{v}$ to $\mathbb{M}$ maps $h$ to $-h$. On the other hand, if $v \in V_{6}$ then the anti-reflection $-R_{v}$ extends to an isometry of $\mathbb{M}$ preserving $h$. This extension is the anti-reflection with respect to the 2-plane generated by $h$ and $v$. In particular, it represent also an element of $\operatorname{Aut}^{+}\left(\mathbb{M}^{0}\right)$.

The union of the mirrors $H_{v}$ for all $v \in V_{2}$ gives after projectivization a union $\mathcal{H}_{\Delta} \subset P\left(\mathbb{M}^{0} \otimes \mathbb{C}\right)$ of hyperplanes, and a similar union of $H_{v}$ for all $v \in V_{6}$ gives another union of hyperplanes, $\mathcal{H}_{\infty} \subset P\left(\mathbb{M}^{0} \otimes \mathbb{C}\right)$.

2.6.1. "Surjectivity" of the period map. The image of the period map per : $\mathscr{C}_{s}^{\sharp} \rightarrow$ $\mathscr{D}$ is the complement of $\mathcal{H}_{\infty}$, and the fibers of per are $P G L(6, \mathbb{C})$ orbits. For a given $p \in \mathscr{D} \backslash \mathcal{H}_{\infty}$, the 2-roots $\delta \in V_{2}$ such that $H_{\delta}$ contains $p$ form an elliptic root system, whose irreducible components are of types $A, D$, and $E$. These components generate the Milnor lattices of the singular points of a cubic with the period $p$.

Remark. The description of the fibers given in the first part of the statement is equivalent to what is called usually the injectivity of the period map (here, at the level of cubics with simple singularities). The second part of the statement includes an intermediate, in some sense, statement that the variations of a singular cubic fourfold contain a simultaneous versal deformation of the singularities if all of them are simple.

2.7. The period map in a real setting ([FK2]). Let us fix a geometric involution $c: \mathbb{M} \rightarrow \mathbb{M}$, see 2.4. A real c-marked nonsingular cubic fourfold (respectively, cubic fourfold with simple singularities) is, by definition, a real non-singular cubic fourfold (respectively, cubic fourfold with simple singularities) equipped with a marking $\phi$ such that $\phi \circ$ conj $^{*}=c \circ \phi$. If such a $c$-marking exists, the cubic fourfold is said to be of homological type $c$.

We denote by $\mathscr{C}_{\mathbb{R}}^{c} \subset \mathscr{C}_{\mathbb{R}}$ (respectively, $\mathscr{C}_{s, \mathbb{R}}^{c} \subset \mathscr{C}_{s, \mathbb{R}}$ ) the set of real nonsingular cubic fourfolds (respectively, cubic fourfolds with simple singularities) of homological type $c$, and by $\mathscr{C}_{\mathbb{R}}^{c \sharp}, \mathscr{C}_{s, \mathbb{R}}^{c \sharp}$ the respective sets of $c$-marked real cubic fourfolds. The two latter sets can be seen as the real parts of $\mathscr{C}^{\sharp}$ and $\mathscr{C}_{s}^{\sharp}$ with respect to the involution which sends $(X, \phi) \in \mathscr{C}^{\sharp}$ to $\left(\operatorname{conj}(X), c \circ \phi \circ\right.$ conj $\left.^{*}\right)$.

Let us extend $c$ to a complex linear involution on $\mathbb{M} \otimes \mathbb{C}$ and denote also by $c$ the induced involutions on $\mathbb{M}^{0} \otimes \mathbb{C}, P=P\left(\mathbb{M}^{0} \otimes \mathbb{C}\right)$, and $\widehat{\mathscr{D}}$. Note that $c$ permutes the two components $\mathscr{D}$ and $\overline{\mathscr{D}}$ of $\widehat{\mathscr{D}}$, and thus, $\bar{c}(\mathscr{D})=\mathscr{D}$, where $\bar{c}: \mathbb{M}^{0} \otimes \mathbb{C} \rightarrow \mathbb{M}^{0} \otimes \mathbb{C}$ is the composition of $c$ with the complex conjugation in $\mathbb{M}^{0} \otimes \mathbb{C}$.

Let $\widehat{\mathscr{D}}_{\mathbb{R}}^{c}$ and $\mathscr{D}_{\mathbb{R}}^{c}$ denote the fixed point set of $\bar{c}$ restricted to $\widehat{\mathscr{D}}$ and $\mathscr{D}$. The second set, $\mathscr{D}_{\mathbb{R}}^{c}$, consists of the lines generated by $w=u_{+}+i u_{-}$such that $u_{ \pm} \in \mathbb{M}_{ \pm}^{0}(c) \otimes \mathbb{R}$, $u_{+}^{2}=u_{-}^{2}<0$, and the orientation $u_{+}, u_{-}$is the prescribed one. Since $c$ is geometric, both $\mathscr{D}_{\mathbb{R}}^{c}$ and its (trivial) double covering $\widehat{\mathscr{D}_{\mathbb{R}}^{c}}$ are nonempty.

As it follows from definitions, the period point of a c-marked real cubic fourfold belongs to $\mathscr{D}_{\mathbb{R}}^{c}=\{x \in \mathscr{D} \mid c(x)=\bar{x}\}$. Therefore, we may speak of a real period map $\operatorname{per}_{\mathbb{R}}: \mathscr{C}_{s, \mathbb{R}}^{c \sharp} \rightarrow \mathscr{D}_{\mathbb{R}}^{c}$ and call $\mathscr{D}_{\mathbb{R}}^{c}$ the real period domain of real c-marked cubic fourfolds.

2.7.1. "Surjectivity" of the period map in a real setting. The image of the real period map $\operatorname{per}_{\mathbb{R}}: \mathscr{C}_{s, \mathbb{R}}^{c \sharp} \rightarrow \mathscr{D}_{\mathbb{R}}^{c}$ is the complement of $\mathcal{H}_{\infty} \cap \mathscr{D}_{\mathbb{R}}^{c}$, and the fibers of $\operatorname{per}_{\mathbb{R}}$ are $P G L(6, \mathbb{R})$ orbits. For a given $p \in \mathscr{D}_{\mathbb{R}}^{c} \backslash \mathcal{H}_{\infty}$, the 2-roots $\delta$ such that $H_{\delta}$ contains $p$ form an elliptic root system invariant under c-action. Its irreducible components which are invariant under c-action generate the Milnor lattices of the real singular points of a cubic with the period $p$. 
As usual, such a real statement can be deduced from the corresponding complex statement, which means here from Theorem 2.6.1. To do it we use the approach applied in ACT in a similar situation.

Proof. The action of $P G L(6, \mathbb{C})$ on $\mathscr{C}_{s}^{\sharp}$ and the map $\mathscr{C}_{s}^{\sharp} / P G L(6, \mathbb{C}) \rightarrow \mathscr{D} \backslash \mathcal{H}_{\infty}$ induced by the period map are proper. Therefore, there is a complete Riemannian metric on $\mathscr{C}_{s}^{\sharp}$ which is invariant under the action of $P G L(6, \mathbb{C})$ and descends to a complete Riemannian metric on $\mathscr{D} \backslash \mathcal{H}_{\infty}$. Taking the average, one can make these metrics invariant under the complex conjugation. Then, the induced Riemannian metric on $\mathscr{C}_{s, \mathbb{R}}^{c \sharp}$ is complete and invariant under the action of $P G L(6, \mathbb{R})$. Since, in addition, the action of $P G L(6, \mathbb{C})$ has finite stabilizers, the map $\mathscr{C}_{s, \mathbb{R}}^{c \sharp} / P G L(6, \mathbb{R}) \rightarrow \mathscr{D}_{\mathbb{R}}^{c}$ (induced by the period map) is proper, which (together with the local Torelli theorem) implies its surjectivity. Its injectivity follows from the local Torelli and the injectivity on the subspace of (marked real) nonsingular cubics.

The second statement is a straightforward consequence of the second statement of Theorem 2.6.1.

\section{Arithmetics ANd topology Under WALl CROSSINGS}

3.1. Indices of the Morse modifications under the facet crossing. Consider non-singular real cubic fourfolds $X$ and $X^{\prime}$ representing adjacent deformation components $C$ and $C^{\prime}$. As we connect $X$ with $X^{\prime}$ by a continuous path which crosses (once) some facet, $\mathcal{F}$, the real locus $X(\mathbb{R})$ experiences a Morse modification of index $0 \leqslant q \leqslant 5$. If we follow this path in the opposite direction, then we observe that $X(\mathbb{R})$ is obtained from $X^{\prime}(\mathbb{R})$ by a Morse modification of index $p=5-q$. In particular, $\chi\left(X^{\prime}(\mathbb{R})\right)-\chi(X(\mathbb{R}))$ is 2 if $q$ is even and -2 if odd. We have also $d\left(X^{\prime}\right)-d(X)= \pm 1$ (see Figure 1 or subsection 3.3 below), where $d$ as before denote the discriminant rank of the eigenlattice $\mathbb{M}_{-}$. If $d\left(X^{\prime}\right)<d(X)$, then we define the index of facet $\mathcal{F}$ as $\operatorname{ind}(\mathcal{F})=q$; in the case $d\left(X^{\prime}\right)>d(X)$ we obtain respectively $\operatorname{ind}(\mathcal{F})=p=5-q$.

\subsubsection{Lemma.}

(1) L-facets have even index and $R$-facets have odd index.

(2) The core sphere of the Morse modification for L-moves and for $R$-moves are null-homologous in $H_{q-1}(X(\mathbb{R}) ; \mathbb{Z} / 2)$.

(3) The core spheres of the Morse modification for $L^{-1}$-moves and for $R^{-1}$-moves are homologically non-trivial in $H_{p-1}(X(\mathbb{R}) ; \mathbb{Z} / 2)$.

Proof. L-moves increase and R-moves decrease the Euler characteristic $\chi(X(\mathbb{R}))$, since $r=\operatorname{rank}\left(M_{-}\right)$plays the role of the horizontal coordinate on Figure 1 (see 2.2). This implies (1). After L-moves and R-moves the discriminant rank (i.e., the vertical coordinate) $d(X)=\frac{1}{2}\left(b_{*}(X)-b_{*}(X(\mathbb{R}))\right)$ is decreasing and thus $b_{*}(X(\mathbb{R}))$ is increasing. Conversely, after $L^{-1}$-moves and $R^{-1}$-moves $b_{*}(X(\mathbb{R}))$ is decreasing. This implies (2) and (3).

\subsubsection{Lemma.}

(1) An R-facet has either index 1, or 3 ;

(2) the L-facets between $C^{0,0}$ and $C_{I}^{1,0}$ have either index 0 , or 4;

(3) all other L-facets have index 2. 
Proof. R-facets cannot have index 5, as follows from the the K4-graph and connectedness of $X(\mathbb{R})$ for $X \notin C_{I}^{1,0}$. This implies (1). A spherical component of $x \in C_{I}^{1,0}$ may be only obtained by a Morse modification of index 0 or 4 , which implies (2).

To prove (3), we observe that if L-facet has index 4, then by 3.1.1(2) its core sphere should be homologically trivial $(\bmod 2)$ and thus the Morse modification increases the number of components of $X(\mathbb{R})$, like in the case of an index 0 modification.

3.2. Cuspidal cubics and their perturbations. In this section we examine cubics of arbitrary dimension $n$.

Assume that a wall separating two deformation components contains two facets adjacent through a cuspidal stratum. Consider a generic point, $\alpha \in \Delta(\mathbb{R})$, of this stratum, which represents a real cubic $X_{\alpha}$ with a cusp and no other singular points. Near the cusp, in a suitable affine chart, the equation of $X_{\alpha}$ is $z^{3}+f_{2}+z g_{2}+f_{3}=0$, where $f_{2}=f_{2}(x, y)=\sum_{i=1}^{p} x_{i}^{2}-\sum_{j=1}^{q} y_{j}^{2}, p+q=n$, and $g_{2}=g_{2}(x, y), f_{3}=f_{3}(x, y)$ are some homogeneous polynomial of degree 2 and 3, respectively. Let us include $X_{\alpha}$ in a twodimensional linear system of cubics $X_{b, c}=\left\{f_{b, c}=0\right\}, f_{b, c}=z^{3}+b z+c+f_{2}+z g_{2}+f_{3}$, $b, c \in \mathbb{R}$. This linear system yields a versal deformation of the cusp singularity, moreover, there exists a local change of $(x, y)$-coordinates reducing, without change of $z$ coordinate, the polynomials $f_{b, c}$ to $z^{3}+b z+c+f_{2}$. It implies that near $\alpha$ our linear system intersects with the discriminant $\Delta$ transversally along a cuspidal curve $\Delta_{b, c}=\left\{4 b^{3}+27 c^{2}=0\right\}$. In particular, near $\alpha$ the hypersurface $\Delta(\mathbb{R})$ is a topological manifold which is split by the cuspidal stratum (corresponding to $b=c=0$ ) into a union of two adjacent facets: one corresponding to $c>0$ and the other to $c<0$.

To treat below the cubics of any dimension we have to extend the notion of index of facets in accordance with our definition for $n=4$. We do it via the following "coorientation" convention: the total $\mathbb{Z} / 2$-Betti number $b_{*}\left(X_{\alpha}(\mathbb{R})\right)$ of the real locus of the cubic representing a generic point, $\alpha \in P_{n, 3}(\mathbb{R})$, in the space of real cubics should increase as $\alpha$ crosses a facet in the positive direction.

This convention does coorient the facets adjacent to the cuspidal strata as it is shown on Figure 3: the normal vectors looks into the "thinner" region bounded by the cusp-shaped discriminant. This is a part of Lemma 3.2.1 below, where we show that such crossing a facet adds a handle to $X_{\alpha}$. For this aim, we look at the part $B_{b, c}=X_{b, c}(\mathbb{R}) \cap B_{\varepsilon}$ of $X_{b, c}$ inside a Milnor ball $B_{\varepsilon}$ (of radius $\varepsilon$ ), where $|b|,|c|<<\varepsilon<<1$.

\subsubsection{Lemma.}

(1) The facet $c>0$ of the discriminant $\Delta_{b, c}=\left\{4 b^{3}+27 c^{2}=0\right\}$, has index $q$, and its facet $c<0$ has index $p$.

(2) If $4 b^{3}+27 c^{2}<0$, then $B_{b, c}$ is a smooth $n$-ball properly embedded in the $(n+1)$ ball $B_{\varepsilon}$.

(3) If $4\left(b^{\prime}\right)^{3}+27\left(c^{\prime}\right)^{2}>0$, then $B_{b^{\prime}, c^{\prime}}$ is isotopic to $B_{b, c} \#\left(S^{p} \times S^{q}\right)$, i.e., ambient connected sum of the above $B_{b, c}$ with an unknotted handle.

Proof. Let us check the base of induction, $n=0$, that is the case of one variable polynomial $z^{3}$ (in this case, $p=q=0$ ). It is sufficient to examine the behavior of $z^{3}-3 t^{2} z+2 t^{3}-\tau$. For $\tau=0$ this polynomial has a double real root $z=t$ and a simple real root $z=-2 t$. If $t>0$, then this double root disappears for $\tau<0$ and turns into two real roots for $\tau>0$, hence, the index of this facet is equal to $0(=q)$. If $t<0$, then the double root disappears for $\tau>0$ and turns into two real roots for $\tau<0$; therefore, to get an increase of the total Betti number (here it is the number of real roots) with 
Figure 3. INDICES OF FACETS ADJACENT

THROUGH A CUSPIDAL STRATUM
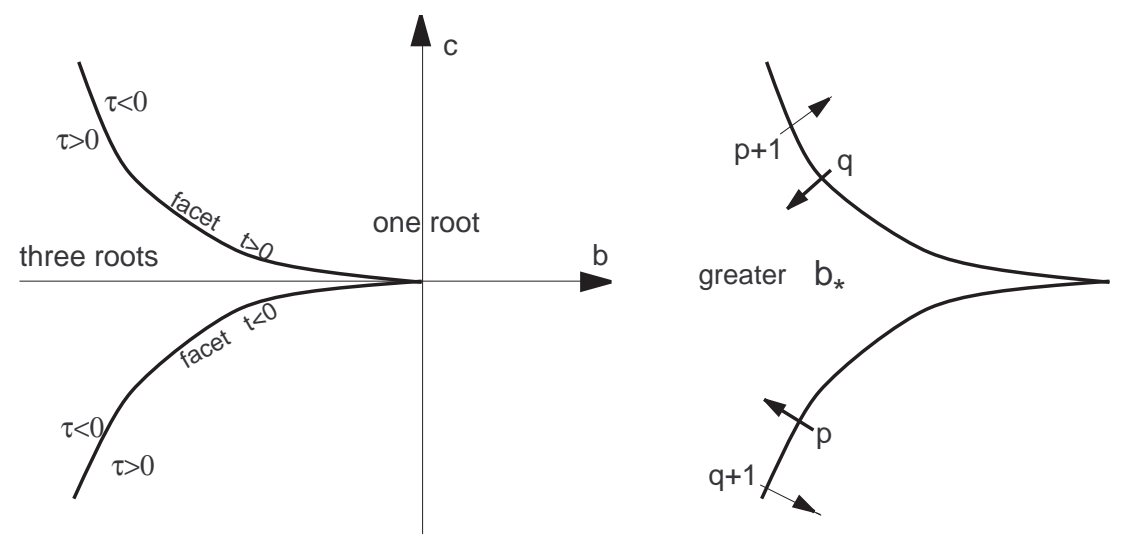

The base case is on the left-hand side figure. Its stabilization is on right-hand side figure, where the arrows show the direction of crossing the facets, and next to the arrow stand the indices of the corresponding Morse modifications.

respect to growing $\tau$ we alternate the sign of the equation, and thus find that the index of this facet is equal to $0(=p)$.

Since the stabilization of the equation (i.e., adding or subtracting the square of a new variable) does not change the total Betti number of its locus in the Milnor ball, it is now straightforward to complete the proof of (1) by induction.

Parts (2) and (3) are also proved by induction. Namely, consider regions $B_{b, c}^{ \pm}=$ $\left\{(x, y, z) \in B_{\varepsilon} \mid \pm f_{b, c} \geqslant 0\right\}$ separated by $B_{b, c}$. After we increase $p$ or $q$, we get a new locus $B_{b, c}$, which is obtained by a suspension, that is can be identified with the "double" of the corresponding region $B_{b, c}^{ \pm}$(the "double" in this context is obtained from two copies of $B_{b, c}^{ \pm}$glued along $\left.B_{b, c}\right)$. This immediately implies (2). For proving (3) we should additionally notice that a handle in the ambient connected sum $B_{b^{\prime}, c^{\prime}}=B_{b, c} \#\left(S^{p} \times S^{q}\right)$ is not knotted provided that the spheres $S^{p} \times \mathrm{pt}$ and $\mathrm{pt} \times S^{q}$ (which are the vanishing cycles corresponding to the facets $c>0$ and $c<0$ respectively) bound disjoint balls (each in the corresponding region $B_{b^{\prime}, c^{\prime}}^{ \pm}$). One can easily see that the suspension (which is increasing $p$ or $q$ ) preserves this property of the spheres.

Returning to dimension $n=4$ we get the following result.

\subsubsection{Corollary.}

(1) In a pair of cuspidally adjacent $R$-facets one has always index 1 and another index 3.

(2) In a pair of cuspidally adjacent $L$-facets separating $C^{0,0}$ and $C_{I}^{1,0}$ one has index 0 and another index 4.

3.3. Arithmetics of the wall-crossing ([FK1, FK2]). Before sticking to the case of cubic fourfolds, let us make a somewhat general remark concerning real non-singular projective hypersurfaces $X$ of any dimension $n$, and their involutions $c: H^{n}(X) \rightarrow$ $H^{n}(X)$ induced by the complex conjugation. It is a well known (and simple) fact 
that adjacency of the deformation components of a pair of such hypersurfaces, $X_{ \pm} \in$ $P_{n, d}(\mathbb{R}) \backslash \Delta(\mathbb{R})$, implies that their involutions, $c_{ \pm}$, are related by the Picard-Lefschetz transformation. Namely, we can connect $X_{ \pm}$by a family $X_{t}$ of non-singular hypersurfaces, $X_{t} \in P_{n, d} \backslash \Delta$, which follows a path in the real parameter space $P_{n, d}(\mathbb{R})$ with exception of an arc, $\gamma$, in the complex domain, $P_{n, d} \backslash P_{n, d}(\mathbb{R})$. Then the composition $c_{-} \circ c_{+}$turns out to be the monodromy along the loop $\bar{\gamma}^{-1} * \gamma$. If $\gamma$ is a small arc which turns around the obstructing facet $\mathcal{F}$ counterclockwise this monodromy is, by definition, the Picard-Lefschetz transformation.

In even dimensions $n$, the Picard-Lefschetz transformation maps $x \in H^{n}\left(X_{+}\right)$to $R_{v}(x)=x-(-1)^{\frac{n}{2}}(v \cdot x) v$ where $v$ is the vanishing (co)-cycle. Therefore, the two involutions $c_{ \pm}$coincide in the orthogonal complement of $v$, while $v$ jumps from \pm 1 eigenspace of $c_{+}$to the opposite $\mp 1$-eigenspace of $c_{-}$. Thus, by the Lefschetz trace formula, $\chi\left(X_{+}(\mathbb{R})\right)-\chi\left(X_{-}(\mathbb{R})\right)$ equals 2 if $v$ belongs to the $(+1)$-eigenspace, and -2 if $v$ belongs to the (-1)-eigenspace. It is also well-known (and straightforward) that the discriminants, $d\left(X_{+}\right)$and $d\left(X_{-}\right)$, of $X_{ \pm}$should differ by 1 . Namely, $d\left(c_{+}\right)-d\left(c_{-}\right)=1$ if $v \cdot x$ is even for all $x$ in the both eigenlattices of $H^{n}\left(X_{+}\right)$, and $d\left(c_{+}\right)-d\left(c_{-}\right)=-1$ otherwise.

In the case of cubic fourfolds, we have shown the converse: the relation $c_{-} \circ c_{+}=R_{v}$ turns out to be a sufficient criterion for (coarse) adjacency of the deformation components (our original argument in FK1] was greatly simplified in [FK2], after Theorem 2.6.1 appeared and Theorem 2.7.1 was derived from it). This can be summarized as follows.

3.3.1. Lemma. Real cubic fourfolds $X_{+}$and $X_{-}$represent adjacent coarse deformation components if and only if there exists a lattice isomorphism $\mathbb{M}\left(X_{+}\right) \rightarrow \mathbb{M}\left(X_{-}\right)$ which preserves the polarization classes $h_{X_{ \pm}} \in \mathbb{M}\left(X_{ \pm}\right)$and identifies the involution $c_{-}$ with the composition $R_{v} \circ c_{+}=c_{+} \circ R_{v}$ for some 2-root $v \in \mathbb{M}_{ \pm}\left(X_{+}\right)$. Moreover, the move leading from the deformation component of $X_{+}$to that of $X_{-}$is

(1) $R$-move, if $v \in \mathbb{M}_{+}\left(X_{+}\right)$and $v \cdot x=0 \bmod 2$ for all $x \in \mathbb{M}_{+}\left(X_{+}\right)$,

(2) L-move, if $v \in \mathbb{M}_{-}\left(X_{+}\right)$, and $v \cdot x=0 \bmod 2$ for all $x \in \mathbb{M}_{-}\left(X_{+}\right)$,

(3) $L^{-1}$-move if $v \in \mathbb{M}_{+}\left(X_{+}\right)$and $v \cdot x=1 \bmod 2$ for some $x \in \mathbb{M}_{+}\left(X_{+}\right)$,

(4) $R^{-1}$-move if $v \in \mathbb{M}_{-}\left(X_{+}\right)$and $v \cdot x=1 \bmod 2$ for some $x \in \mathbb{M}_{-}\left(X_{+}\right)$.

Now let us apply Theorem 2.7.1 to the cuspidal cubics similarly.

As it follows from analysis of the local model performed in 3.2 , there exist two ways of perturbation of a real cuspidal hypersurface $X_{0}$ (of any dimension $n$ ): one perturbation does not change topologically its real locus, i.e., yields $X_{+}$with $X_{+}(\mathbb{R})$ homeomorphic to $X_{0}(\mathbb{R})$, and the other perturbation adds a handle (of an appropriate index) to $X_{0}(\mathbb{R}) \cong$ $X_{+}(\mathbb{R})$, and in particular, yields $X_{-}$with $b_{*}\left(X_{-}(\mathbb{R})\right)=b_{*}\left(X_{+}(\mathbb{R})\right)+2$ (or equivalently, $\left.d\left(X_{-}\right)=d\left(X_{+}\right)-1\right)$. We explained also that the latter perturbation shifts $X_{0}$ from a cuspidal stratum of $\Delta(\mathbb{R}) \subset P_{n, d}(\mathbb{R})$ inside the thin region of the cuspidal slice of $\Delta(\mathbb{R})$ shown on Figure 3.

Analysis of the same local model $\sum_{i=1}^{p} x_{i}^{2}-\sum_{j=1}^{q} y_{j}^{2}+z^{3}+b z+c$ of a cuspidal perturbation shows that for even $n=p+q$, the involution $c_{-}: H^{n}\left(X_{-}\right) \rightarrow H^{n}\left(X_{-}\right)$acts in the Milnor lattice $A_{2}$ of the cusp as $(-1)^{q}$. In the other words, $A_{2}$ is embedded into the corresponding eigenlattice. (Note that the involution $c_{+}$in $H^{n}\left(X_{+}\right)$interchanges some pair of roots, $v_{1}, v_{2}$, generating $A_{2}$.) As it follows from Theorem 2.7.1, such $A_{2}$-sublattice should not contain 6-roots (since otherwise the corresponding periods, namely the intersection of mirrors $H_{\delta}$ over generating 2-roots of $A_{2}$, would lie in $\mathcal{H}_{\infty}$ ). 
In the case of cubic fourfolds, existence of such appropriate $A_{2}$-sublattice yields an arithmetical criterion allowing to detect cuspidal strata on the boundary of a deformation component of real cubic fourfolds.

3.3.2. Lemma. Consider the wall $\mathcal{W}$ separating a pair of adjacent components represented by cubics $X_{ \pm}$. Assume that $d\left(X_{+}\right)>d\left(X_{-}\right)$. Then $\mathcal{W}$ contains a cuspidal stratum if and only if

(1) there exist 2-roots $v_{1}, v_{2} \in \mathbb{M}_{-}\left(X_{-}\right)$such that

$$
v_{1} \cdot v_{2}=-1 \text { and }\left(v_{1}-v_{2}\right) \cdot \mathbb{M}_{-}\left(X_{-}\right) \neq 0 \quad \bmod 3, \quad \text { if } \mathcal{W} \text { is } R \text {-wall; }
$$

(2) there exist 2-roots $v_{1}, v_{2} \in \mathbb{M}_{+}^{0}\left(X_{-}\right)$such that

$$
v_{1} \cdot v_{2}=-1 \text { and }\left(v_{1}-v_{2}\right) \cdot \mathbb{M}_{+}^{0}\left(X_{-}\right) \neq 0 \quad \bmod 3, \quad \text { if } \mathcal{W} \text { is } L \text {-wall. }
$$

Proof. Any $A_{2}$-lattice contains precisely six elements of square six, namely, $\pm\left(v_{1}-v_{2}\right)$, $\pm\left(2 v_{1}+v_{2}\right)$, and $\pm\left(v_{1}+2 v_{2}\right)$, where $v_{1}, v_{2}$ are 2-roots forming a basis. The two latter pairs of vectors are congruent modulo 3 to the first pair (since, $\left(2 v_{1}+v_{2}\right)+\left(v_{1}-v_{2}\right)=$ $3 v_{1}$, etc.). Therefore, under the hypothesis that $\left(v_{1}-v_{2}\right) \cdot \mathbb{M}^{0}\left(X_{-}\right) \neq 0 \bmod 3$, a sublattice $A_{2}$ does not contain 6-roots and so, by Theorem 2.7.1 a generic point in $\mathcal{D} \cap\left\{w \mid w \cdot v_{1}=w \cdot v_{2}=0\right\}$ (and, as a consequence, a generic real point in this intersection) does not belong to $\mathcal{H}_{\infty}$. Since $A_{2}$ is a sublattice of $\mathbb{M}_{-}\left(X_{-}\right)$(of $\mathbb{M}_{+}^{0}\left(X_{-}\right)$) in the case of an $R$-move (respectively, $L$-move), the condition $\left(v_{1}-v_{2}\right) \cdot \mathbb{M}^{0}\left(X_{-}\right) \neq 0$ $\bmod 3$ can be equally stated as is done in the Lemma.

3.3.3. Corollary. Each of the R-walls, except the ones which lead to $C^{10,1}$ or $C_{I}^{2,1}$, has a cuspidal stratum.

Proof. To apply Lemma 3.3 .2 we need to embed suitably $A_{2}$ to $\mathbb{M}_{-}\left(X_{-}\right)$. According to Tables 2 and 3 , in each of the corresponding cases the lattice $\mathbb{M}_{-}\left(X_{-}\right)$contains as a direct summand either $\langle 2\rangle \oplus U$, or $D_{4}$, or $E_{7}$, or $E_{8}$. In $D_{4}, E_{7}$, and $E_{8}$ we may take any standard embedding of $A_{2}$. In $\langle 2\rangle \oplus U$ we pick $v_{1}=e-u_{1}$ and $v_{2}=u_{1}+u_{2}$, where $e$ is a generator of $\langle 2\rangle$ and $u_{1}, u_{2}$ are standard generators of $U$.

\section{RAMIFIED CONNECTED SUMS}

4.1. Double ramified coverings and their Morse modifications. Assume that $U$ is a compact $n$-manifold and $L$ a codimension two submanifold coming transversely to $\partial U$ along its boundary $\partial L \subset \partial U$. Recall that double coverings $\pi: \widetilde{U} \rightarrow U$ ramified along $L$ are classified up to isomorphism by the characteristic class $w_{1} \in H^{1}(U \backslash L ; \mathbb{Z} / 2)$ of the restriction of $\pi$ over $U \backslash L$ (that is of the unramified part of $\pi$ ). Given an element $w \in H^{1}(U \backslash L ; \mathbb{Z} / 2)$, there exist a covering ramified along $L$ with the characteristic class $w_{1}=w$ if and only if the coboundary map $H^{1}(U \backslash L ; \mathbb{Z} / 2) \rightarrow H^{2}(U, U \backslash L ; \mathbb{Z} / 2)$ sends $w$ to the Thom class of the normal bundle of $L$. As is known, the latter condition is equivalent to a possibility to realize the class dual to $w_{1}$ in $H_{n-1}(U, \partial U \cup L ; \mathbb{Z} / 2)$ by a codimension one compact submanifold $F \subset U$ whose boundary splits as $\partial F=L \cup F^{\partial}$, where $F^{\partial}=\partial F \cap \partial U$, so that $\partial L=\partial F^{\partial}$ and $F$ is transversal to $\partial U$ along $F^{\partial}$. In constructions we allow $F$ and $F \cap \partial U$ to have real algebraic singularities outside $L$ (then still by duality, $F$ defines a class $w_{1}$ as above; in fact, Morse singularities are sufficient for our purposes) and call such $F$ a characteristic hypersurface of the ramified 
covering $\pi$ or a characteristic membrane bounding $L$. For simplicity, we suppose from now on that $L$ and $F^{\partial}$ are disjoint parts of $\partial F$.

It may be worth recalling that given a non-singular characteristic hypersurface $F$, a representative, $\pi: \widetilde{U}^{F} \rightarrow U$, of the associated class of ramified covering is obtained from a disjoint union $U \sqcup U$ of two copies of $U$ by cutting them along $F$ and then gluing together along $F$ in a cross-like fashion, so that each side of $F$ in one copy of $U$ is identified with the opposite side of $F$ in the other copy. Note that $F$ does not need to be orientable, and it is sufficient that "the sides" are just locally defined.

We need to treat the coverings in one-parameter families. More precisely, to compare coverings of $U$ with coverings of $U_{\bullet}=U \times[0,1]$, and vice versa. To include a covering of $U$ into a covering of $U_{\bullet}$ it is necessary and sufficient to extend $L$ to a proper codimension two submanifold $L_{\bullet}$ of $U_{\bullet}$ and $w_{1}$ to a proper class of its complement. It is for this task that characteristic hypersurfaces are especially convenient: given an extension $L \bullet$ of $L$ it is sufficient to extend $F$ to a characteristic membrane $F_{\bullet}$ bounding $L_{\bullet}$.

In what follows we are concerned exclusively with a particular situation where the projection to $[0,1]$ restricted to $L_{\bullet}$ is a Morse function, so that we may consider $L$ • as a special family $L_{t}, L_{\bullet}=\cup_{t \in[0,1]} L_{t} \times t$, such that $L_{t}$ is a smooth isotopy which experiences ambient Morse modifications at finitely many points. As to $F_{\bullet}$, we restrict ourselves to continuous families, $F_{\bullet}=\cup_{t} F_{t}$, where $F_{t}$ is a characteristic membrane of $L_{t}$ for each noncritical value of $t$. (Recall that smoothness of $F_{\bullet}$ is not required, and moreover, it can be even not a topological submanifold: it is enough that it gives a homology cycle.)

It is transparent that such a construction leads to diffeomorphisms $\widetilde{U}^{F_{0}} \rightarrow \widetilde{U}^{F_{t}}$, if $L_{t}$ is an isotopy. It is also a well-known fact in the knot theory, that a Morse modification of index $q$ performed on $L_{t}$ yields a Morse modification of index $q+1$ on $U^{F_{t}}$. Still, we sketch the proof below, since we could not find a reference suitable for our needs.

4.1.1. Lemma. (1) If $L_{t}$ is an isotopy, then the family $F_{t}$ yields a continuous family of diffeomorphisms $\widetilde{\phi}_{t}: \widetilde{U}^{F_{0}} \rightarrow \widetilde{U}^{F_{t}}$. If $F_{t}^{\partial}=\varnothing$ for all $t \in[0,1]$, then such a family $\widetilde{\phi}_{t}$ is identical on $\partial \widetilde{U}^{F_{t}}=\partial U \sqcup \partial U$.

(2) Assume that there is only one critical value $t \in(0,1)$, so that $L_{1}$ is obtained from $L_{0}$ by a Morse modification of index $q$. Then $\widetilde{U}^{F_{1}}$ is obtained from $\widetilde{U}^{F_{0}}$ by a Morse modification of index $q+1$.

Proof. An isotopy of the branching locus $L_{t}$ in (1) can be extended to an ambient isotopy $\phi_{t}: U \rightarrow U$. The latter lifts to a family of diffeomorphisms $\widetilde{\phi}_{t}: \widetilde{U}^{F_{0}} \rightarrow \widetilde{U}^{F_{t}}$, since the characteristic classes $w_{1}$ of all the coverings do match. If $F_{t}^{\partial}=\varnothing$, then we may choose $\phi_{t}$ identical on $\partial U$ and its lifting $\widetilde{\phi}_{t}$ identical on $\partial U \sqcup \partial U$.

To prove (2), it is sufficient to analyze a local model of an elementary cobordism of index $q$. Namely, it is enough to consider the case of $U=D^{n}$ (n-ball), where $L \subset$ $U \times[0,1]$ is $(n-1)$-ball, and $L_{t}$ represent a Morse modification of index $q$ inside $U \times[0,1]$. Such elementary cobordism connects standard framed spheres, $L_{0}=D^{p} \times S^{q-1} \subset U \times 0$ and $L_{1}=S^{p-1} \times D^{q}, p+q=n-1$, on the bottom and the top of the cylinder $U \times[0,1]$, and is restricted to $\partial U \times[0,1]$ as a product-cobordism, $\partial L_{t}=S^{p-1} \times S^{q-1} \subset \partial U \times t$. Passing to the double covers over $U \times t$ ramified along $L_{t}$, we observe an index $q+1$ model cobordism between $D^{p} \times S^{q} \times D^{1}$ and $S^{p} \times D^{q} \times D^{1}$.

4.2. Ramified connected sums. Assume that $F^{\partial}=\varnothing$ and $U$ is embedded into each of two closed $n$-manifolds $X_{1}$ and $X_{2}$. Then we can remove $U$ from each of $X_{i}$ and glue instead $\widetilde{U}^{F}$ via the identity map of the boundary $\partial \widetilde{U}^{F}=\partial U \sqcup \partial U \subset\left(X_{1} \backslash U\right) \cup\left(X_{2} \backslash U\right)$. 
The new manifold will be denoted $X_{1} \#^{F} X_{2}$ and called the ramified connected sum of $X_{1}$ and $X_{2}$. It is easy to see that $X_{1} \#^{F} X_{2}$ depends only on the embedding of $F$ into $X_{1}$ and $X_{2}$, but not on the particular choice of its neighborhoods $U$. Moreover, if we consider a continuous family $F_{t}$ like in Lemma 4.1.1, then we can apply it to obtain the following.

4.2.1. Corollary. (1) If $L_{t}=\partial F_{t}$ is an isotopy, then there is a continuous family of diffeomorphisms $\psi_{t}: X_{1} \#^{F_{0}} X_{2} \rightarrow X_{1} \#^{F_{t}} X_{2}$.

(2) Assume that there is only one critical value $t \in(0,1)$, so that $L_{1}$ is obtained from $L_{0}$ by a Morse modification of index $q$. Then $X_{1} \#^{F_{1}} X_{2}$ is obtained from $X_{1} \#^{F_{0}} X_{2}$ by a Morse modification of index $q+1$.

In what follows we will need the following particular example. Assume that $F^{\prime}=$ $F \cup T$, where $T$ is a "solid torus" $D^{p} \times S^{q-1}$ embedded into some $n$-ball $D^{n} \subset U \backslash F$, $p+q=n$.

4.2.2. Lemma. If $T$ is unknotted in $D^{n}$, then $X_{1} \#^{F^{\prime}} X_{2}$ is diffeomorphic to a connected sum of $X_{1} \#^{F} X_{2}$ with $\left(S^{1} \times S^{n-1}\right) \#\left(S^{p} \times S^{q}\right)$.

Proof. The double covering over $S^{n}$ ramified along an unknotted $S^{p-1} \times S^{q-1}$ is wellknown to be $S^{p} \times S^{q}$. Note that $X_{1} \#^{F^{\prime}} X_{2}$ is obtained from $X_{1} \#^{F} X_{2}$ after removing a pair of balls (pull-back of $D^{n}$ ) and gluing instead the double covering over $D^{n}$ ramified along $\partial T$, which is diffeomorphic to $S^{p} \times S^{q}$ with a pair of balls removed. The onehandle involved is orientable (since ramified coverings preserve orientability), and thus we just take a connected sum of $X_{1} \#^{F} X_{2}$ with $\left(S^{1} \times S^{n-1}\right) \#\left(S^{p} \times S^{q}\right)$.

4.3. Perturbation of the union of a quadric with a hyperplane. Here we describe topologically the result of perturbation of the union $P \cup Q \subset \mathbb{R} P^{n+1}$ of a real hyperplane $P$ and a real quadric $Q$.

Let $\left[x_{0}: \ldots x_{n}: y\right]$ denote homogeneous coordinates in $\mathbb{R} \mathrm{P}^{n+1}$ and $P=\mathbb{R} \mathrm{P}^{n}$ be defined by $y=0$. We assume that $Q$ is defined by equation $f_{2}-\varepsilon y^{2}=0$ where $f_{2}=f_{2}\left(x_{0}, \ldots, x_{n}\right)$ is a non-degenerate quadratic form and $\varepsilon>0$ is a fixed parameter. The intersection $V=P \cap Q=\left\{f_{2}(x)=0\right\}$ gives a splitting $P=P_{+} \cup P_{-}$, where $P_{ \pm}=\left\{[x] \in P \mid \pm f_{2}(x) \geqslant 0\right\}$. If $f_{2}$ has signature $(p, q), p+q=n+1$, then $P_{+}$is a tubular neighborhood of $\mathbb{R P}^{p-1}$ and $P_{-}$the tubular neighborhood of a complementary $\mathbb{R P}^{q-1}$. Forgetting the coordinate $y$ gives the projection $p: Q \rightarrow P_{+}$which is obtained from the orientation double covering $\widetilde{p}: \widetilde{P}_{+} \rightarrow P_{+}$by identifying the pairs of points $\widetilde{p}^{-1}(x)$ along the boundary.

4.3.1. Lemma. The locus $F_{+}=P_{+} \cap\{f=0\}$ of any non-singular polynomial $f$ of odd degree is a characteristic hypersurface of the double covering $\widetilde{p}: \widetilde{P}_{+} \rightarrow P_{+}$.

Proof. An odd degree hypersurface $\{f=0\}$ in $P$ is dual to the generator of $H^{1}(P ; \mathbb{Z} / 2)$ and so is characteristic for the orientation covering $\widetilde{P} \rightarrow P$. Restricting the latter over $P_{+}$we conclude that $F_{+}$is characteristic for $\widetilde{P}_{+} \rightarrow P_{+}$.

By a perturbation of $P \cup Q$ via $f_{3}=f_{3}\left(x_{0}, \ldots, x_{n}, y\right)$ we will mean a real cubic $Z$ defined by $y\left(f_{2}-\varepsilon y^{2}\right)+\delta f_{3}=0$, where $0<\delta<<\varepsilon$. Such a cubic is non-singular provided that $P, Q$ and $f_{3}=0$ (or equivalently, $f_{2}(x)=0$ and $f_{3}(x, 0)=0$ inside $P$ ) intersect transversally.

Denote this intersection by $L$ and assume the above transversality. Then, the intersection of $\left\{f_{3}=0\right\}$ with $P_{+}$is a characteristic membrane bounding $L$. 
Consider the double $D\left(P_{+}\right)$of $P_{+}$, which is obtained from two copies of $P_{+}$by gluing them together along the boundary $V$. Let $U$ denote a neighborhood of $P_{+}$obtained by adding to it a collar $V \times[0,1)$ of its boundary, so that $F \subset P_{+} \subset \operatorname{Int}(U)$. Note that $P_{+}$lies both in $P$ and in $D\left(P_{+}\right)$, and we may extend these embeddings of $P_{+}$to embeddings $U \subset P$ and $U \subset D\left(P_{+}\right)$.

Our next target is the following proposition, which will play a key role in the forthcoming analysis of cubic fourfolds.

4.3.2. Proposition. If the quadratic form $f_{2}(x)$ is non-degenerate and the intersection of $f_{2}(x)=0$ and $f_{3}(x, 0)=0$ in $P$ is transversal, then for any $0<\delta<<\varepsilon$ the real locus $Z(\mathbb{R})$ of the cubic $Z=\left\{y\left(f_{2}-\varepsilon y^{2}\right)+\delta f_{3}=0\right\}$ is diffeomorphic to $P \#^{F} D\left(P_{+}\right)$.

4.4. The perturbation neck. We start proving Proposition 4.3 .2 with checking certain properties of the perturbations of $P \cup Q \subset \mathbb{R P}^{n+1}$ in a somewhat more general setting, assuming that $P$ and $Q$ are transverse real non-singular hypersurfaces defined by polynomials $f$ and $g$ of degrees $\ell$ and $m$. As before, we let $V=P \cap Q$ and denote by $Z \subset \mathbb{R P}^{n+1}$ the result of a perturbation described by a polynomial $f g+\delta h$, $0<\delta<<1$, assuming in addition that $P, Q$, and $h=0$ intersect transversally. Thus, their intersection, which we denote $L$, is non-singular.

It is well known (and trivial) that topologically a small perturbation of $P \cup Q$ is localized in a tubular neighborhood $N \subset \mathbb{R}^{n+1}$ of $V$. Namely, the tubular neighborhoods $N_{P}=N \cap P$ and $N_{Q}=N \cap Q$ of $V$ are removed from $P$ and $Q$ and are replaced by a certain perturbation neck $N_{P, Q} \subset N$, which has also a projection $p_{P, Q}: N_{P, Q} \rightarrow V$. Namely, the fibers $I_{v}^{P}$ and $I_{v}^{Q}$ of the projections $N_{P} \rightarrow V$ and $N_{Q} \rightarrow V$ over $v \in V$ look topologically like line segments intersecting in the middle points. The cross-like fiber $I_{v}^{P} \cup I_{v}^{Q}$ of $(P \cup Q) \rightarrow V$ is perturbed into a fiber of $p_{P, Q}$, which looks generically as a pair of $\operatorname{arcs} I \sqcup I$ connecting the two endpoints $\partial I_{s}^{P}$ with the two endpoints $\partial I_{s}^{Q}$, and the two possible ways of such connection alternate as we cross the locus $L=V \cap\{h=0\}$. Over $L$ the fibers of $p_{P, Q}$ are non-generic, namely, they remain $I_{v}^{P} \cup I_{v}^{Q}$.

Figure 4. FACTORIZATION OF A PERTURBATION NECK

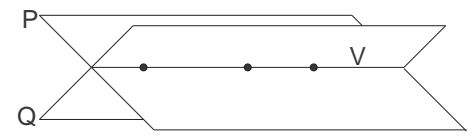

a) Transversal hypersurfaces

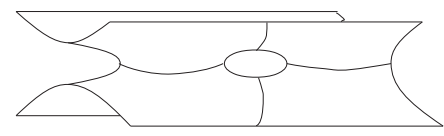

b) Perturbation neck
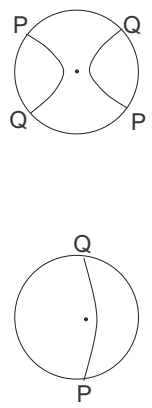

(1)

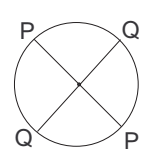

c) Fibers

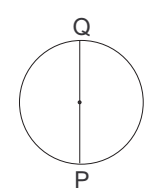

d) Quotients
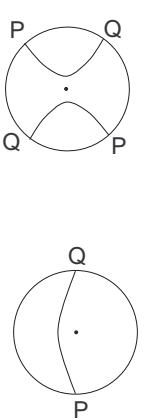

a) Hypersurfaces $P \cup Q$, where $L \subset V=P \cap Q$ is marked by dots. b) The corresponding perturbation neck $N_{P, Q}$. c) Fibers of $p_{P, Q}: N_{P, Q} \rightarrow V$. The central singular fiber $I_{v}^{P} \cup I_{v}^{Q}$ is over $v \in L$; it is perturbed in two ways as $v$ shifts from $L$. d) Passing to the quotient by $\tau$ (central symmetry) makes all the fibers homeomorphic to $I$. 
Next we observe existence of an involution $\tau: N_{P, Q} \rightarrow N_{P, Q}$ which preserves the fibers and act on each fiber as the central symmetry. The quotient space $N_{P, Q} / \tau$ is fibred over $V$ trivially with a fiber $I$, and so can be identified with $V \times[-1,1]$. This implies that the quotient map $q: N_{P, Q} \rightarrow V \times[-1,1]$ has to be a ramified covering.

4.4.1. Lemma. The quotient map $q: N_{P, Q} \rightarrow V \times[-1,1]$ is a double covering ramified along $L \times 0$.

Let us choose the identification $N_{P, Q} / \tau=V \times[-1,1]$ so that $\partial N_{P} / \tau$ and $\partial N_{Q} / \tau$ are identified with $V \times-1$ and $V \times 1$ respectively.

The lemma below describes the characteristic hypersurface $F$ of the double covering $q$ under an additional assumption that one of the degrees, $m=\operatorname{deg}(Q)$, is even. This assumption implies that $V$ is co-orientable in $P$ and thus its tubular neighborhood $N_{P}$ can be identified with $N_{P, Q} / \tau=V \times[-1,1]$.

4.4.2. Lemma. Assume that $m$ is even. Then the intersection $F=(V \times[0,1]) \cap$ $\{h=0\}$ is the characteristic hypersurface of the ramified covering $q$, with respect to the identification of the quotient space $N_{P, Q} / \tau$ with $N_{P}=V \times[-1,1]$, as described above.

Proof. Note that $q$ has the ramification locus $L=F \cap(V \times 0)=\{f=g=h=0\}$, as required by Lemma 4.4.1. The restriction of $q$ over $V \times-1$ is trivial which corresponds to the characteristic cycle $\varnothing=F \cap(V \times-1)$. This implies that $V \times[0,1] \cap\{h=0\}$ is the characteristic cycle of $q$ because $H^{1}(V \times[-1,1], V \times-1 ; \mathbb{Z} / 2)=0$.

Now, fixing $f$ and $g$ let us assume that $h$ varies continuously so that $L$ experiences a single Morse modification.

4.4.3. Corollary. If $h$ varies so that $L$ experiences a Morse modification of index $q$, then $Z$ experiences a Morse modification of index $q+1$.

Proof. The pieces $P \backslash N_{P}$ and $Q \backslash N_{Q}$ do not change in process of variation of $Z$, and the perturbation neck $N_{P, Q}$ experiences a Morse modification of index $q+1$, as follows from Lemmas 4.4 .2 and 4.1 .1 .

4.5. Proof of Proposition 4.3.2. According to 4.4, a perturbation of $P \cup Q$ gives $Z=\left(P \backslash N_{P}\right) \cup\left(Q \backslash N_{Q}\right) \cup N_{P, Q}$, whereas $P \#^{F} D\left(P_{+}\right)=(P \backslash U) \cup\left(D\left(P_{+}\right) \backslash U\right) \cup \widetilde{U}^{F}$. Note that $P \backslash N_{P}$ splits into a pair of components, namely, $P \backslash U$ and $D\left(P_{+}\right) \backslash U$, which are diffeomorphic to $P_{-}$and $P_{+}$respectively. So, the proof of Proposition 4.3.2 is reduced to the following statement.

4.5.1. Lemma. The part $\left(Q \backslash N_{Q}\right) \cup N_{P, Q} \subset Z$ is diffeomorphic to $\widetilde{U}^{F}$.

Proof. Recall that $U$ is $P_{+}$enlarged with a collar in $P_{-}$, so we can take $U=\left(P_{+} \backslash\right.$ $\left.N_{P}\right) \cup N_{P}$.

Then $\widetilde{U}^{F}$ splits into a union of two pieces, one of which is the pull-back of $N_{P}$ and the pull-back of $\mathrm{Cl}\left(P_{+} \backslash N_{P}\right)$. Lemma 4.4.2 says that the first piece is just $N_{P, Q}$. The other piece is obviously diffeomorphic to the pull-back of $P_{+}$. It can be identified with $\widetilde{P}_{+}$, because of Lemma 4.3.1. To complete the proof it is left to recall that $Q \backslash N_{Q}$ is diffeomorphic to $\widetilde{P}_{+}$.

4.6. Perturbation of nodal cubics. Like in 4.3, let us consider a hyperplane $P=$ $\{y=0\}$ and a quadric $Q=\left\{f_{2}(x)=\varepsilon y^{2}\right\}$ in $\mathbb{R P}^{n+1}$ with coordinates $x=\left(x_{0}, \ldots, x_{n}\right)$ and $y$, where $f_{2}$ is a real non-degenerate quadratic form. But now let us choose a cubic form $f_{3}=f_{3}(x)$ independent of $y$. Suppose like before that the intersection 
$Y=\left\{f_{2}=f_{3}=0\right\}$ is transversal (then $Y$ is a non-singular $(n-1)$-fold), and denote by $X_{\varepsilon, \delta}$ the cubic $n$-fold $\left\{y\left(f_{2}-\varepsilon y^{2}\right)+\delta f_{3}=0\right\}$. In 4.3, under the assumption that $0<\delta<<\varepsilon$, we treated $X_{\varepsilon, \delta}(\mathbb{R})$ as a perturbation of $P \cup Q$. One can also view $X_{\varepsilon, \delta}$ as a perturbation of a nodal cubic $\left\{y f_{2}+\delta f_{3}=0\right\}$ supposing that $0<\varepsilon<<\delta$. The following Lemma shows, in particular, that both perturbations yield the same deformation type.

4.6.1. Lemma. For any $f_{2}(x), f_{3}(x)$ like above, there exists $\varkappa>0$ such that for any choice of positive $\varepsilon, \delta<\varkappa$ the locus $X_{\varepsilon, \delta}=\left\{y\left(f_{2}(x)-\varepsilon y^{2}\right)+\delta f_{3}(x)=0\right\}$ in $\mathbb{R P}^{n+1}$ is non-singular.

Proof. Under the quasi-homogeneous change of variables $(x, y) \mapsto\left(t x, t^{-2} y\right)$ the polynomial $y\left(f_{2}(x)-\varepsilon y^{2}\right)+\delta f_{3}(x)$ is transformed into

$$
t^{-2} y\left(f_{2}(t x)-\varepsilon\left(t^{-2} y\right)^{2}\right)+\delta f_{3}(t x)=y\left(f_{2}(x)-\varepsilon t^{-4} y^{2}\right)+\delta t^{3} f_{3}(x),
$$

so that the plane of parameters $\varepsilon, \delta$ becomes foliated by curves $\varepsilon^{3} \delta^{4}=$ const each representing projectively equivalent $X_{\varepsilon, \delta}$. Hence, the statement follows from the nonsingularity of $X_{1, \delta}$ for small $\delta>0$.

From now on we stick to the case of our interest, $n=4$.

4.6.2. Proposition. Consider a vertex $v_{K 3}$ of the K3-graph and a vertex $v_{K 4}$ of the K4-graph which correspond to each other. Assume that a non-degenerate quadric $\left\{f_{2}=\right.$ $0\}$ and a cubic $\left\{f_{3}=0\right\}$ intersect transversally, their intersection $\left\{f_{2}=f_{3}=0\right\}$ represents the vertex $v_{K 3}$, and the sign of $f_{2}$ is chosen so that $\chi\left(P_{+}\right)=1$, where $P_{+}=\left\{x \in P \mid f_{2}(X) \geqslant 0\right\}$. Then there exists $\varkappa>0$ such that for any choice of positive $\varepsilon, \delta<\varkappa$ the cubic fourfold $X_{\varepsilon, \delta}=\left\{y\left(f_{2}-\varepsilon y^{2}\right)+\delta f_{3}=0\right\}$ is non-singular and represent vertex $v_{K 4}$.

Proof. The nodal cubic fourfolds $X_{0, \delta}$ defined by $y f_{2}+\delta f_{3}$ are in central projection correspondence with $Y$ and represent an edge in the K4-graph. By theorem 2.5.1, it is the leftmost endpoint (providing a smaller value of the coordinate $r$ ) of the edge which corresponds to the vertex $v_{K 3}$ representing $Y$. The two endpoints are given by two perturbations, $\left\{y\left(f_{2}-\varepsilon y^{2}\right)+\delta f_{3}\right\}$ and $\left\{y\left(f_{2}+\varepsilon y^{2}\right)+\delta f_{3}\right\}$, of $X_{0, \delta}$. The relation $2 r=b_{*}(X)-\chi(X(\mathbb{R}))-4$ (see 2.2) implies that the vertex $v_{K 4}$ is represented by that perturbation which yields a cubic fourfold with a greater value of $\chi(X(\mathbb{R}))$. As it follows from Proposition 4.3.2, $\chi\left(X_{\varepsilon, \delta}(\mathbb{R})\right)=\chi(P)+2 \chi\left(P_{+}\right)-\chi(L)$, which is greater than the Euler characteristic for another perturbation, $\chi(P)+2 \chi\left(P_{-}\right)-\chi(L)$, since by our assumption $\chi\left(P_{+}\right)=1$ and thus $\chi\left(P_{-}\right)=0$.

Finally, we apply Lemma 4.6.1.

4.6.3. Corollary. Under the assumption of Proposition 4.6.2, the real locus of a cubic fourfold of the type $v_{K 4}$ is diffeomorphic to $P \#^{F} D\left(P_{+}\right)$, where $F=\left\{x \in P_{+} \mid f_{3}(x)=\right.$ $0\}$.

Proof. Choosing $\delta<<\varepsilon$ we can view $Y_{\varepsilon, \delta}$ as a perturbation of $\left\{y\left(f_{2}-\varepsilon y^{2}\right)\right\}$ and apply Proposition 4.3.2.

Let us fix $f_{2}$ and continuously vary $f_{3}$, so that the real K3-surfaces $Y_{t}$, defined by $f_{3, t}=f_{2}=0, t \in[-1,1]$, are non-singular for $t \neq 0$, and $Y_{0}$ is nodal, so that $Y_{ \pm t}$, where $t>0$, represent a pair of adjacent vertices $v_{K 3}^{ \pm}$of the K3-graph. Let $X_{ \pm}$be cubic fourfolds representing the corresponding vertices $v_{K 4}^{ \pm}$of the K4-graph. 
4.6.4. Proposition. If $Y_{t}(\mathbb{R}), t<0$, experiences at $t=0$ a Morse modification of index $q$, then $X_{+}(\mathbb{R})$ is obtained from $X_{-}(\mathbb{R})$ by a Morse modification of index $q+1$.

Proof. According to Corollary 4.6.3, we have $X_{ \pm}(\mathbb{R})=P \#^{F_{ \pm}} D\left(P_{+}\right)$, where $F_{ \pm}=$ $P_{+} \cap\left\{f_{3, \pm 1}=0\right\}$. Now it remains to apply Corollary 4.2.1.

\section{Proof of the MAIN THEOREM}

5.1. The proof in the general case. We have already proved Theorem 1.2.1 in the cases $C_{I}^{1,0}$ and $C^{0,0}$, see 2.3. As the next step, let us consider the real cubic fourfolds, $X$, representing the vertices of $\Gamma_{K 4}$ which are obtained from $C^{0,0}$ by L-moves (i.e., lying on the upper-left side of $\left.\Gamma_{K 4}\right)$.

5.1.1. Lemma. If a cubic $X$ represents $C^{i, 0}, i \geqslant 0$, or $C_{I}^{i, 0}, i>1$, then $X(\mathbb{R})$ is diffeomorphic to $\mathbb{R} \mathrm{P}^{4} \# i\left(S^{2} \times S^{2}\right)$.

Proof. We prove this lemma by induction in $i$. The base case, $i=0$, is already established in Corollary 2.3.2. Consider a cubic fourfold $X^{\prime}$ representing $C^{i-1,0}, i>0$, and assume that $X$ represents either $C^{i, 0}$ or $C_{I}^{i, 0} \neq C_{I}^{1,0}$. By Lemma 3.1.2 $(3), X(\mathbb{R})$ is obtained from $X^{\prime}(\mathbb{R})$ by attaching a 2-handle, and by Lemma 3.1.1 (2) the core circles of this handle is null-homologous. By inductive assumption, $X^{\prime}(\mathbb{R})=\mathbb{R} \mathrm{P}^{4} \#(i-1)\left(S^{2} \times S^{2}\right)$ has $\pi_{1}=\mathbb{Z} / 2$, and thus the core circle is contractible. Attaching of a 2-handle along a contractible circle is equivalent to taking a connected sum either with $S^{2} \times S^{2}$, or with $\mathbb{C P}^{2} \# \overline{\mathbb{C P}}$, depending on the framing of the 2-handle. The second option is impossible because all cubic fourfolds have $w_{2}(X(\mathbb{R}))=0$, and thus, the orientation covering space of $X_{\mathbb{R}}$ must have even intersection form.

Our next aim is to apply $R$-moves to the cubics treated above.

5.1.2. Lemma. If the $R$-wall separating the cubics $X^{\prime}$ of type $C^{i, j-1}, j \geqslant 1$, from the cubics $X$ of type $C^{i, j}$ or $C_{I}^{i, j}$ contains a cuspidal stratum, then $X_{\mathbb{R}}$ is diffeomorphic to $X_{\mathbb{R}}^{\prime} \#\left(S^{1} \times S^{3}\right)$.

Proof. By corollary 3.2.2(1), one of the facet-strata adjacent to the cuspidal stratum has index 1 , and so $X(\mathbb{R})$ is obtained by adding a 1-handle to $X^{\prime}(\mathbb{R})$. The latter is connected by Lemma 2.3.1 and Corollary 2.3.2. It is also non-orientable, so, adding 1-handle means taking a connected sum with $S^{1} \times S^{3}$.

This allows us to deduce the main theorem 1.2.1 in all but a few cases.

5.1.3. Corollary. If a cubic $X$ belongs to the type $C^{i, j}$ or $C_{I}^{i, j}$ different from $C_{I}^{1,0}$, $C^{10,1}$, and $C_{I}^{2,1}$, then $X_{\mathbb{R}}$ is diffeomorphic to $\mathbb{R P}^{4} \# i\left(S^{2} \times S^{2}\right) \# j\left(S^{1} \times S^{3}\right)$.

Proof. Lemma 5.1 .1 covers the cases with $j=0$. Vertices $C^{i, j}$ and $C_{I}^{i, j}, j>0$ can be reached from $C^{i, 0}$ by R-moves. According to Corollary 3.3.3, the assumption of Lemma 5.1 .2 is satisfied unless $X$ belongs to the type $C^{10,1}$, or $C_{I}^{2,1}$. So, applying Lemma 5.1 .2 $j$ times we obtain the given description of $X(\mathbb{R})$. (Note that the exceptional vertices are terminal and thus are not obstacles in the sequence of R-moves.)

5.2. The case of $C_{I}^{2,1}$. Like in 4.3, we start with a perturbation of a reducible real cubic fourfold $P \cup Q$, where quadric $Q$ is defined by $f_{2}-\varepsilon y^{2}$. Now we specify $f_{2}$ so that the region $P_{+}$is a tubular neighborhood of $P^{2}(\mathbb{R})=\left\{\left[x_{0}: x_{1}: x_{2}: 0: 0\right]\right\} \subset P$, for example, we may take $f_{2}=x_{0}^{2}+x_{1}^{2}+x_{2}^{2}-x_{3}^{2}-x_{4}^{2}$. As a perturbation term $f_{3}$ we pick 
a degree 3 homogeneous polynomial in three variables $x_{0}, x_{1}, x_{2}$ (thus, independent of $\left.x_{3}, x_{4}, y\right)$ such that the curve defined in $P^{2}(\mathbb{R})$ by equation $f_{3}=0$ is non-singular, which insures in particular the transversality of intersection between $f_{3}=0$ and $f_{2}=0$ in $P^{5}(\mathbb{R})$.

Note that $F=P_{+} \cap\left\{f_{3}=0\right\}$ is the pull-back of this curve under the tubular neighborhood projection $q: P_{+} \rightarrow P^{2}(\mathbb{R})$ (that is the projection which forgets the coordinates $\left.x_{3}, x_{4}\right)$. Therefore, under above choices, $F$ is diffeomorphic to the product of the curve by $D^{2}$.

A real plane cubic curve may have one or two connected components, and we need to consider the both possibilities. Let us denote by $f_{3}^{(k)}, k=1,2$, a polynomial defining a real nonsingular cubic curve with $k$ component. Then, $F^{(k)}=P_{+} \cap\left\{f_{3}^{(k)}=0\right\}$ becomes a solid torus for $k=1$ and a pair of solid tori for $k=2$.

Following Section 4.3, consider real cubic fourfolds $X^{(k)}(k=1,2)$ defined by equation $y\left(f_{2}-\varepsilon y^{2}\right)+\delta f_{3}^{(k)}=0$, where we pick positive $\varepsilon$ and $\delta$ to be smaller than the constant $\varkappa$ provided by Lemma 4.6.1.

5.2.1. Lemma. $X^{(1)}$ and $X^{(2)}$ belong to the types $C^{1,0}$ and $C_{I}^{2,1}$ respectively.

Proof. The real locus of the K3-surface $f_{2}=f_{3}^{(k)}=0$ is $\partial F^{(k)}$, that is $k$ copies of a torus. Such a K3-surface is known to be of type $C^{1,0}$ if $k=1$ and $C_{I}^{2,1}$ if $k=2$ (see for example the survey $[\mathrm{DK})$. Since $\chi\left(P_{+}\right)=1$, Proposition 4.6 .2 implies that the cubic fourfold $X^{(k)}$ belongs to the corresponding type, as is stated.

5.2.2. Lemma. $X^{(2)}(\mathbb{R})=X^{(1)}(\mathbb{R}) \#\left(S^{2} \times S^{2}\right) \#\left(S^{1} \times S^{3}\right)$.

Proof. One of the real components of the two-component cubic curve $f_{3}^{(2)}=0$ is non contractible and isotopic to a real line; the other component bounds a disc, $D \subset P^{2}(\mathbb{R})$. Let $T_{\infty}, T_{0}$ denote the corresponding solid torus components of $F^{(2)}$. The component $T_{0}$ is an unknotted torus contained in the 4 -disc $q^{-1}(D)$ disjoint from $T_{\infty}$. On the other hand, the only real component of the curve $f_{3}^{(1)}=0$ is isotopic in $P^{2}(\mathbb{R})$ to a real line, and therefore $F^{(1)}$ is isotopic to $T_{\infty}$. So, we can apply Lemma 4.2 .2 to compare $X^{(2)}=P \#^{F^{(2)}} D\left(Q^{+}\right)$with $X^{(1)}=P \#^{F^{(1)}} D\left(Q^{+}\right)$and to conclude that $X^{(2)}(\mathbb{R})=$ $X^{(1)}(\mathbb{R}) \#\left(S^{2} \times S^{2}\right) \#\left(S^{1} \times S^{3}\right)$.

5.2.3. Corollary. If a cubic $X$ belongs to the type $C_{I}^{2,1}$, then $X(\mathbb{R})$ is diffeomorphic to $\mathbb{R} \mathrm{P}^{4} \# 2\left(S^{2} \times S^{2}\right) \#\left(S^{1} \times S^{3}\right)$.

Proof. According to Lemma 5.2 .1 the cubic $X^{(1)}$ belongs to type $C^{1,0}$. So, by Lemma 5.1.1, its real part $X^{(1)}(\mathbb{R})$ is diffeomorphic to $\mathbb{R} P^{4} \#\left(S^{2} \times S^{2}\right)$. Thus, it remains to apply Lemma 5.2.2.

\subsection{The case of $C^{10,1}$.}

5.3.1. Lemma. There exist a real non-degenerate homogeneous quadratic polynomial $f_{2}=f_{2}\left(x_{0}, \ldots, x_{4}\right)$ and a continuous family of real cubic homogeneous polynomials $f_{3, t}=f_{3, t}\left(x_{0}, \ldots, x_{4}\right), t \in[-1,1]$, such that the complete intersections $Y_{t} \subset P^{4}, f_{2}=$ $f_{3, t}=0$, are K3-surfaces which are non-singular of type $C^{10,0}$ for $t<0$, and nonsingular of type $C^{10,1}$ for $t>0$, while $Y_{0}$ has a nodal singularity.

Proof. According to the K3-graph, the 6-polarized K3-surfaces of type $C^{10,0}$ are adjacent to the K3-surfaces of type $C^{10,1}$. Therefore, we can find a path which leads from one component to another and intersects the wall between them at its non-singular 
point. Let $f_{2, t}=f_{3, t}=0, t \in[-1,1]$, be such a path, so that $t=0$ corresponds to a nodal K3-surface. For a generic choice of such a path the quadric $f_{2,0}$ is nonsingular and, thus, the quadrics $f_{2, t}$ are also non-singular for sufficiently small $|t|>0$. Recall that deformation equivalent real quadrics are actually projective equivalent, so, applying suitable projective transformations we can turn $f_{2, t}$ into a constant family of quadrics, $f_{2}$.

The topological types of $X(\mathbb{R})$ for K3-surfaces $X$ are well known (see, for example, the survey [DK]). In particular, in the case of K3-surfaces of types $C^{10,0}$ and $C^{10,1}$, the locus $X(\mathbb{R})$ is homeomorphic respectively to $S_{10}$ and $S_{10} \sqcup S^{2}$, where $S_{10}$ stands for an orientable surface of genus 10. Note also that a Morse modification of a surface which brings about a new component $S^{2}$ can be either of index 0 (a birth of sphere), or 2 (splitting off a sphere from another component). Let us show that in our case the second possibility is not realizable.

5.3.2. Lemma. Assume that $Y_{t}, t \in[-1,1]$ is a family of real K3-surfaces which represent type $C^{10,0}$ for $t<0$, type $C^{10,1}$ for $t>0$, and experience a Morse modification at $t=0$. Then the Morse modification is of index 0 .

Proof. The component $S^{2}$ of $Y_{1}(\mathbb{R})$ represents a class $s$ in the K3-lattice $\mathbb{L}=H_{2}\left(Y_{1}\right)$. This class belongs to the eigen-sublattice $\mathbb{L}_{+}$since $S^{2}$ is $c$-invariant, and it has square $s^{2}=-2$ since the normal bundle to the real locus is isomorphic to the cotangent bundle. The vanishing cycle $v \in \mathbb{L}$ of the degeneration $Y_{1} \rightarrow Y_{0}$ also has square $v^{2}=-2$ and, since the Morse index is even, the vanishing cycle also belongs to $\mathbb{L}_{+}$. If $Y_{t}(\mathbb{R})$ experiences a Morse modification of index 2, then $s \cdot v= \pm 1$, so that $s$ and $v$ span a sublattice $A_{2}$ in $\mathbb{L}_{+}$. On the other hand, the lattice $\mathbb{L}_{+}$is isomorphic to $U$ and, hence, does not contain $A_{2}$.

5.3.3. Corollary. The wall separating the deformation components $C^{10,0}$ and $C^{10,1}$ in the space of real cubic fourfolds contains a facet of index 1.

Proof. Consider a family of real cubic fourfolds $X_{t}$ obtained by perturbation of $P \cup Q$, where $P=P^{4}(\mathbb{R})$ and $Q=\left\{f_{2}-y^{2}=0\right\}$, with a perturbation term $f_{3, t}$ given by Lemma 5.3.1. Namely, the family defined by $y\left(f_{2}-y^{2}\right)+\delta f_{3, t}$ for a suitable $0<\delta<<1$. By Lemma 5.3.2, the family $Y_{t}(\mathbb{R})$ experiences a Morse modification of index 0 at $t=0$. Proposition 4.6.4 implies that $X_{t}(\mathbb{R})$ experiences a Morse modification of index 1 .

5.3.4. Corollary. If a cubic $X$ belongs to type $C^{10,1}$, then $X(\mathbb{R})$ is diffeomorphic to $\mathbb{R P}^{4} \# 10\left(S^{2} \times S^{2}\right) \#\left(S^{1} \times S^{3}\right)$.

Proof. The class $C^{10,0}$ has a representative $X^{\prime}$ with $X^{\prime}(\mathbb{R})=\mathbb{R} \mathrm{P}^{4} \# 10\left(S^{2} \times S^{2}\right)$ as we know from Corollary 5.1.3. By Corollary 5.3.3, our $X(\mathbb{R})$ can be obtained from $X^{\prime}(\mathbb{R})$ by adding 1-handle, i.e., by taking connected sum with $S^{1} \times S^{3}$.

\section{CONCluding REMARKS}

6.1. Topology of cubic threefolds. For non-singular real cubic threefolds $X$ representing seven deformation classes among the eight existing classes with connected real part $X(\mathbb{R})$, the topological type of $X(\mathbb{R})$ was determined by V. Krasnov [Kr2]. He proved that for all of them the real part is diffeomorphic to a connected sum of $\mathbb{R P}^{3}$ with some number of $S^{1} \times S^{2}$. Here, we show that the situation is different for the remaining deformation class, that is $\mathscr{B}(1)_{I}^{\prime}$ in Krasnov's notation. 
To describe the topological type of $X(\mathbb{R})$ for cubic threefolds of class $\mathscr{B}(1)_{I}^{\prime}$, we start with a construction of $X(\mathbb{R})$ following the lines of Section 4.3. Namely, we consider the cubics $X=X_{\varepsilon, \delta}=\left\{y\left(f_{2}-\varepsilon y^{2}\right)+\delta f_{3}=0\right\}$ obtained by a perturbation of $P \cup Q$, where $P=\mathbb{R} \mathrm{P}^{3}$ is a hyperplane $y=0$ in $\mathbb{R} \mathrm{P}^{4}$ with coordinates $\left[x_{0}: x_{1}: x_{2}: x_{3}: y\right]$, and $Q \subset \mathbb{R P}^{4}$ is a real non-singular quadric threefold, $\left\{f_{2}-\varepsilon y^{2}=0\right\}$, such that intersection $V=P \cap Q$ is transversal. In addition, we suppose that the quadric surface $V \subset \mathbb{R} P^{3}$ is a hyperboloid and denote by $\ell_{1}, \ell_{2}$ two real lines representing the two families of real generators of this hyperboloid. Topologically, $V$ is a torus which splits $P$ into a pair of solid tori $P_{ \pm}=\left\{ \pm f_{2} \geqslant 0\right\}$ with the meridians $m_{ \pm}$homologous to $\left[\ell_{1}\right] \pm\left[\ell_{2}\right] \in H_{1}(V)$. Let us orient $V$ and $\ell_{i}$ so that $\ell_{1} \circ \ell_{2}=1$ and consider the curve $L=\left\{f_{2}=f_{3}=0\right\}$ on torus $V$ (as in Section 4.3, we suppose that the quadric $f_{2}=0$ and the cubic $f_{3}=0$ are transversal to each other). Bézout theorem implies that $L$ is homologous to $p\left[\ell_{1}\right]+q\left[\ell_{2}\right] \in H_{1}(V)$ with $p, q \in\{ \pm 1, \pm 3\}$, and one can easily realize all such pairs of $p$ and $q$ by suitable choices of $f_{3}$. In particular, we may consider $[L]=\left[\ell_{2}\right]-3\left[\ell_{1}\right]$, represented by a spiral-shaped single-component curve $L$.

6.1.1. Theorem. Let $V$ and $L$ be as above. Assume that $L$ is homologous to $\left[\ell_{2}\right]-$ $3\left[\ell_{1}\right] \in H_{1}(V)$. Then $X=\left\{y\left(f_{2}-\varepsilon y^{2}\right)+\delta f_{3}=0\right\}$ represents the deformation class $\mathscr{B}(1)_{I}^{\prime}$ and its real part $X(\mathbb{R})$ is the Seifert manifold whose link diagram is the rightmost diagram on Figure 6.

In the Figures and in the proof, we follow traditional Kirby calculus notation and terminology, see [Ki.

Proof. Let $T_{1}$ denote an abstract solid torus bounded by $V$ with the meridian $\ell_{1}$. Since $m_{+} \circ \ell_{1}=-1$, the union $P_{+} \cup T_{1}$ (with the common boundary $V$ ) forms a 3 -sphere. We orient $S^{3}$ so that the inherited orientation on $P_{+}$restricts to the given one on $V$.

Figure 5. RAmified COVERING ALONG $L$

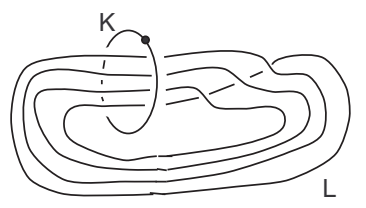

a

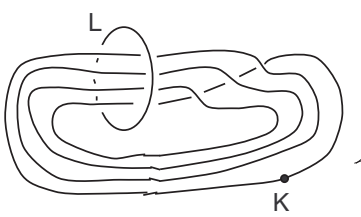

b

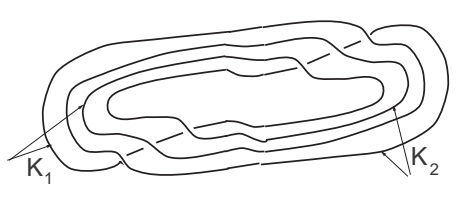

C

On Figure 5a the core circle of $T_{1}$ is presented by the dotted unknot $K$. The exterior of $K$ presents $P_{+}$, where $L$ looks like a $(4,1)$-torus knot, because a positive basis of $V$ is formed by classes $\left[m_{+}\right]$and $-\left[\ell_{1}\right]$, and $[L]=\left[\ell_{2}\right]-3\left[\ell_{1}\right]=\left[m_{+}\right]-4\left[\ell_{1}\right]$.

Note that $m_{-}$can be seen as a $(2,1)$-torus knot, since $\left[m_{-}\right]=-\left[m_{+}\right]+2\left[\ell_{1}\right]$. The latter gives framing $(-2)$ to $K$, which means that $P$ is obtained from our 3 -sphere by a Dehn $(-2)$-surgery along $K$. Note also that the double $D\left(P_{+}\right)$is obtained by 0 -surgery along $K$.

Furthermore, the Seifert surface $F=P_{+} \cap\left\{f_{3}=0\right\}$ is disjoint from $K$, which implies that for a neighborhood $U \supset P_{+}$chosen like in 1.3 , our ramified double covering $\widetilde{U}^{F} \rightarrow$ $U$ can be extended to the (unique) double covering $\pi: M_{L} \rightarrow S^{3}$ ramified along $L$, where $M_{L}$ it also a 3 -sphere, since $L$ is an unknot. This implies that $X(\mathbb{R})=P \#^{F} D\left(P_{+}\right)$is obtained from $M_{L}$ by a Dehn surgery along the 2-component link $K_{1} \cup K_{2}=\pi^{-1}(K)$ with certain framings $n_{1}, n_{2}$ inherited from the framings -2 and 0 of $K$ in $S^{3}$. 
Note that $K$ and $L$ can be interchanged by an isotopy, and the diagram on Figure $5 \mathrm{~b}$ presents the same link $K \cup L$. Now $K$ looks like a $(4,1)$-torus knot, and its pre-image in $M_{L}=S^{3}$ can be seen on Figure $5 \mathrm{c}$ as a $(4,2)$-torus link, whose components $K_{1}, K_{2}$ are $(2,1)$-torus knots. The pull-back of the torus framing of $K$ gives the torus framing on each of $K_{i}$. Since the torus framing of a $(p, q)$-torus knot is $p q$, we see that a framing $n$ of $K$ gives framings $(n-2)$ on $K_{i}$, and thus $n_{1}=-4$ and $n_{2}=-2$. So $X(\mathbb{R})$ can be described by the leftmost link diagram on Figure 6.

Figure 6. Framed Link Diagrams of $X(\mathbb{R})$

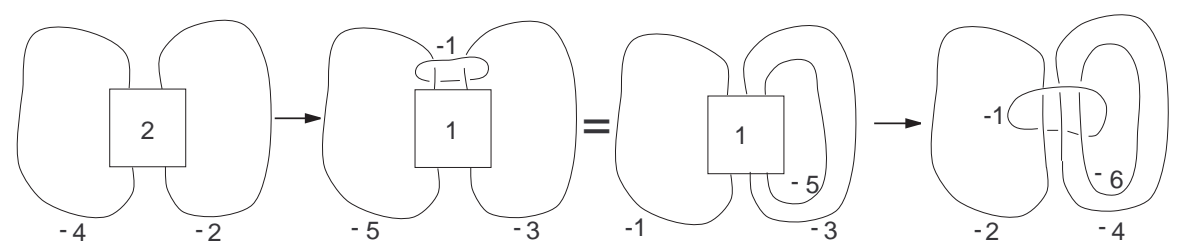

The components $K_{1}$ and $K_{2}$ can be unlinked by a blowup move (a Kirby move introducing an additional (-1)-components), as is shown on Figure 6, and we obtain after another blowup move the rightmost link diagram, which describes a Seifert 3manifold.

The 3-manifold $X(\mathbb{R})$ is obviously connected and is not homeomorphic to $\mathbb{R} \mathrm{P}^{3}$ with handles: it can be distinguished for instance by its fundamental group $\pi_{1}(X(\mathbb{R}))=$ $\left\langle a, b, c \mid a^{2}=b^{4}=c^{6}=a b c\right\rangle$, and even by its homology $H_{1}(X(\mathbb{R}))=\mathbb{Z} / 2+\mathbb{Z} / 2$ (see for example the presentation of $\pi_{1}$ given in $[\mathrm{O}$ ). So, the deformation class of our cubic $X$ differs from the ones analyzed in [Kr2], and so has to be the class $\mathscr{B}(1)_{I}^{\prime}$.

Remark. Note that Figure 6 describes the link of the singularity $x y\left(x^{3}+y^{2}\right)+z^{2}=$ 0 known as a weighted homogeneous singularity of type $Z_{12}$. It is one of Arnold's fourteen exceptional singularities of modality one (its Dolgachev numbers $(p, q, r)$ and the Gabrielov numbers $\left(p^{\prime}, q^{\prime}, r^{\prime}\right)$ both are equal to $\left.(2,4,6)\right)$.

6.2. Cubics-handles in higher dimensions. In higher dimensions, to construct non-singular real $n$-fold cubics $X \subset P^{n+1}$ one may follow the same scheme as in the proof of the main theorem: start with the case $X(\mathbb{R})=\mathbb{R} \mathrm{P}^{n}$ and then make it "develop" and acquire more and more various handles due to crossing of the facets of the discriminant by special moves in the parameter space. Here, let us indicate a few first steps on this way.

According to Lemma 2.3.1, the cubics $X$ with disconnected real part (which implies $X(\mathbb{R})=\mathbb{R P}^{n} \sqcup S^{n}$ ) form a single deformation component, furthermore, this deformation component, which we denote below by $C_{0}$, has only one adjacent component, which is formed by cubics $X$ with $X(\mathbb{R})=\mathbb{R P}^{n}$. Let us call the latter component root component and denote it $C_{\text {root }}$. A special role of the root component is due to the following property.

6.2.1. Lemma. For any $n \geqslant 0$ and any real non-singular homogeneous cubic polynomial $f(x)=f\left(x_{0}, \ldots, x_{n}\right)$, the real cubic $n$-fold $X \subset P^{n+1}$ defined by equation $z^{3}=f(x)$ belongs to the deformation component $C_{\text {root }}$.

Proof. First, note that $X(\mathbb{R})$ is diffeomorphic to $\mathbb{R P}^{n}$, a diffeomorphism being given by projection $P^{n+1} \rightarrow P^{n}$ forgetting the $z$-coordinate. Moreover, the same projection provides a homeomorphism between $X(\mathbb{R})$ and $\mathbb{R P}^{n}$ even if we authorize $f=0$ to have 
arbitrary singularities. Combining these observations with Lemma 3.2.1, we conclude that the cubic $z^{3}=f_{t}(x)$ does not change the deformation component, when $f_{t}$ crosses a facet of the discriminant. To see that this unique deformation component is $C_{\text {root }}$, it is sufficient to apply Lemma 3.2 .1 to, say, $f=x_{0}\left(x_{1}^{2}+\cdots+x_{n}^{2}\right)+x_{1}^{3}+\cdots+x_{n}^{3}$; then one of the two perturbations described in Lemma 3.2.1 gives $\mathbb{R} \mathrm{P}^{n} \sqcup S^{n}$, while the other contains elements from $C_{\text {root }}$.

6.2.2. Proposition. The boundary of the component $C_{\text {root }}$ contains certain facets $\mathcal{F}_{q}$ with $0 \leqslant q \leqslant n$ such that: $q$ is the index of the facet $\mathcal{F}_{q}$; the facet $\mathcal{F}_{q}$ is adjacent to $\mathcal{F}_{p}$ through a cuspidal stratum if $p+q=n$; and the component $C_{q}=C_{p}$ adjacent to $C_{\text {root }}$ through $\mathcal{F}_{q}$ and $\mathcal{F}_{p}$ is formed by cubics $X$ with $X(\mathbb{R})$ diffeomorphic to $\mathbb{R P}^{n} \#\left(S^{p} \times S^{q}\right)$.

Proof. If $f(x)=0$ is a nodal $(n-1)$-fold cubic, the $n$-fold cubic $z^{3}=f(x)$ belongs to a cuspidal stratum of $\Delta(\mathbb{R}) \subset P_{n, 3}(\mathbb{R})$. It remains, therefore, to apply Lemma 3.2.1 selecting a suitable polynomial $f$ for each pair $p, q$.

The same argument can be used to construct non-singular real cubic $n$-folds whose real part is diffeomorphic to $\mathbb{R P}^{n}$ with several handles.

6.2.3. Proposition. For any integer $k, 0 \leqslant k<\frac{1}{2}(n+1)$, there exists a non-singular real cubic $n$-fold $X \subset P^{n+1}$ with $X(\mathbb{R})$ diffeomorphic to $\mathbb{R P}^{n} \# m_{k}\left(S^{k} \times S^{n-k}\right)$, where $m_{k}$ is equal to $\left(\begin{array}{c}n+1 \\ k\end{array}\right)$. Such a cubic $X$ can be obtained by perturbation of a certain real cubic $X_{0}$ with $m_{k}$ real cusps of index $(k, n-k)$ and with $X_{0}(\mathbb{R})=\mathbb{R P}{ }^{n}$.

Proof. Let us start with a real cubic $(n-1)$-fold defined in $\mathbb{R P}^{n}$ by a cubic polynomial $f_{t}(x)=t\left(x_{0}^{3}+\cdots+x_{n}^{3}\right)-\left(x_{0}+\cdots+x_{n}\right)^{3}$. For $t=(n+1-2 k)^{2}$ with $0 \leqslant k<\frac{1}{2}(n+1)$, such a cubic has $m_{k}$ nodes of index $(k, n-k)$.

The real cubic $n$-fold $X_{0}$ defined in $\mathbb{R} \mathrm{P}^{n+1}$ by the suspension polynomial $f_{t}(x)-z^{3}$ has cusps at the corresponding points, and $X_{0}(\mathbb{R})=\mathbb{R P}^{n}$. As in Lemma 3.2.1, the perturbation $f_{t}(x)-z^{3}-b z-c$ provides, for suitable $b$ and $c$, a real cubic $n$-fold $X$ with $X(\mathbb{R})=\mathbb{R P}^{n} \# m_{k}\left(S^{k} \times S^{n-k}\right)$.

A different kind of examples is generated by cubics with two nodes of different indices. Namely, consider $P^{n+2}$ with homogeneous coordinates $x=\left(x_{0}, \ldots, x_{n}\right), y_{1}, y_{2}$, and a cubic $V$ defined by $F(x, y)=f(x)+y_{1} g_{1}(x)+y_{2} g_{2}(x)+y_{1} y_{2} h(x)$, where $g_{i}$ are quadratic forms and $f, h$ are cubic and linear forms respectively. In the chart $y_{1} \neq 0$ centered at $\left(x, y_{2}\right)=(0,0)$, the affine equation of $V$ is $y_{2} h+g_{1}+\left(f+y_{2} g_{2}\right)$, and, thus, we have a node at the origin if the quadratic form $y_{2} h+g_{1}$ is non-degenerate. Similarly, in the chart $y_{2} \neq 0$ centered at $\left(x, y_{1}\right)=(0,0)$ we have a double point governed by the quadratic form $y_{1} h+g_{2}$. It is easy to choose $g_{i}$ and $h$ so that the two nodes have arbitrary prescribed indices in the range between 1 and $n+1$. Then $F(x, y)-z^{3}$ defines a cubic with two cusps which can be perturbed into handles of the corresponding indices, so that we obtain the following result.

6.2.4. Proposition. For any $n \geqslant 0$ and $1 \leqslant a, b \leqslant n+1$, there exists a real nonsingular cubic $(n+2)$-fold, $X \subset P^{n+3}$, with $X(\mathbb{R})$ diffeomorphic to $\mathbb{R P}^{n+2} \#\left(S^{a} \times\right.$ $\left.S^{n+2-a}\right) \#\left(S^{b} \times S^{n+2-b}\right)$.

6.3. Homological and topological types of higher dimensional cubics. An important consequence of deformation classification of real cubic fourfolds in [FK1] is their homological quasi-simplicity. By definition, this means that the triple $\left(\mathbb{M}(X), c_{X}, h_{X}\right)$ is a complete invariant of a non-singular cubic fourfold $X$ up to coarse deformation equivalence. The same is true for $n$-dimensional cubics $X$ if $n<4$. However, in higher 
dimensions it is no longer true. We show it below restricting ourselves to the case of even $n$ : in this case we do not need to involve any techniques essentially different from what is already discussed in this paper.

The only new ingredient we use in the proof below is a property of algebraic varieties of type I known as Klein's principle (see [K] ): "varieties of type I do not admit development". According to a modern interpretation (see $[\mathrm{R}]$ and $[\mathrm{Vi}]$ ), this refers to the local maximality of such varieties, namely: a real algebraic variety of type I cannot increase its total Betti number $b_{*}$ after crossing the wall in the parameter space. In the case of cubics of even dimension, the proof is easy and can be based on two observations: first, $d=\frac{1}{2}\left(b_{*}(X)-b_{*}(X(\mathbb{R}))\right)$ where, as usual, $d$ is the rank of the 2-periodic discriminant group $\mathbb{M} /\left(\mathbb{M}_{+}+\mathbb{M}_{-}\right)=\mathbb{M}_{ \pm}^{*} / \mathbb{M}_{ \pm}$; second, to decrease $d$ the vanishing cycle $v$ of the wall crossing should be of the form $x \pm c x$, which is incompatible with $v^{2}= \pm 2$ and $x \cdot c x+x^{2}=0 \bmod 2$ (the latter is the definition of type I, see 2.4).

6.3.1. Proposition. For any even $n \geqslant 6$ there exists a pair of real non-singular cubic $n$-folds $X, X^{\prime} \subset P^{n+1}, i=1,2$, which are homologically equivalent but have nonhomeomorphic real point sets (and thus, $X$ is not coarse deformation equivalent to $X^{\prime}$ ).

Proof. Consider representatives $X_{p}$ of the deformation components $C_{p}$ provided by Proposition 6.2.2, $p=0, \ldots, n$. The parity of $p$ determine the rank of each of the lattices $\mathbb{M}_{ \pm}$, and thus, determine their signature (since one of the inertia indices is fixed). The discriminant forms have the same rank (it is less by one than the maximal rank achieved for $C_{\text {root }}$ ) and the same Arf-Brown invariant. Calculation shows that these lattices are indefinite, and thus, according to Nikulin's results (see $[\mathbf{N} \mid$ ), there may be only two isomorphism classes, which correspond to cubics of type I and II, respectively.

On the other hand, our construction of $X(\mathbb{R})=\mathbb{R} \mathrm{P}^{n} \# m_{1}\left(S^{1} \times S^{n-1}\right)$ and $X(\mathbb{R})=$ $\mathbb{R P}^{n} \# m_{3}\left(S^{3} \times S^{n-3}\right)$, where $m_{1}=n+1$ and $m_{3}=\left(\begin{array}{c}n+1 \\ 3\end{array}\right)$, shows that the cubics $X_{p}$ admit a "development", so according to Klein's principle they are all of type II.

Now we may conclude that, for example, the cubics $X_{1}$ and $X_{3}$ have isomorphic eigenlattices, hence they are homologically equivalent. But at the same time, for $n \geqslant 6$, $X_{1}(\mathbb{R})=\mathbb{R} \mathrm{P}^{n} \#\left(S^{1} \times S^{n-1}\right)$ and $X_{3}=\mathbb{R P}^{n} \#\left(S^{3} \times S^{n-3}\right)$ are not homeomorphic.

6.4. Higher dimensional cubics as ramified connected sums. According to Lemma 4.6.1, whatever is the dimension, any family of non-singular cubics $X_{t}$ degenerating to a nodal cubic $X_{0}$ can be transformed into a family of cubics $X_{t}^{\prime}$, such that $X_{t}$ is projectively equivalent to $X_{t}$, and $X_{t}^{\prime}$ degenerates to a cubic splitting into a union of a quadric and a hyperplane. This remarkable property of cubics implies that all the deformation classes of non-singular real cubics can be obtained by small perturbation of such reducible cubics. More precisely, the following statement holds (and follows easily from Lemma 4.6.1 and the possibility to degenerate any non-singular cubic to a nodal one).

6.4.1. Proposition. Any deformation class of non-singular cubic hypersurfaces in $P^{n+1}$, with coordinates $x_{0}, \ldots, x_{n}, y$, admits a representative defined by equation $\left\{y\left(f_{2}-\right.\right.$ $\left.\left.y^{2}\right)+\delta f_{3}=0\right\}$, where $f_{2}=f_{2}(x)=\sum_{i=0}^{n} \pm x_{i}^{2}$ (for a certain choices of signs \pm ), $0<\delta<<1$, and $f_{3}=f_{3}(x, y)$ is some cubic transversal to the quadric $f_{2}$ in the hyperplane $y=0$.

Combining this statement with Proposition 4.3.2, we obtain the following description of topology of real loci. 
6.4.2. Corollary. The real point set $X(\mathbb{R})$ of any non-singular real cubic hypersurface $X \subset P^{n+1}$, for any $n>0$, is diffeomorphic to a ramified connected sum $P \#^{F} D\left(P_{+}\right)$, where $P=P^{n}(\mathbb{R}), D\left(P_{+}\right)$is the double of $P_{+}=\left\{x \in P \mid f_{2} \geqslant 0\right\}$, and $F=\{x \in$ $\left.P_{+} \mid f_{3}=0\right\}$. Here $f_{2}, f_{3}$ are respectively quadratic and cubic forms in $P$, such that $f_{2}$ is non-degenerate and has a transverse intersection with $f_{3}$.

\section{REFERENCES}

[ACT] D. Allcock, J. Carlson, and D. Toledo, Hyperbolic geometry and moduli of real cubic surfaces, arXiv:0707.1058 (to appear in Ann. Sci. ENS).

[DK] A. Degtyarev and V. Kharlamov, Topological properties of real algebraic varieties : du côtè de chez Rokhlin, Uspekhi Mat. Nauk. 55 (2000), no. 4, 129-212.

[FK1] S. Finashin, V. Kharlamov, Deformation classes of real four-dimensional cubic hypersurfaces, J. Alg. Geom. 17 (2008), 677-707.

[FK2] S. Finashin, V. Kharlamov, On the deformation chirality of real cubic fourfolds, arXiv:0804.4882.

[Ki] R. Kirby, A Calculus for Framed Links in $S^{3}$, Invent. Math. 45 (1978), 35-56.

[Kl] F. Klein, Gesammelte mathematische Abhandlungen, Berlin, vol. 2, 1922.

[Kr1] V. Krasnov, Rigid isotopy classification of real three-dimensional cubics, Izvestiya: Mathematics 70 (2006), no. 4, 731-768.

[Kr2] V. Krasnov, On the topological classification of three-dimensional real cubics (to appear in Izvestiya: Mathematics).

[La] R. Laza, The moduli space of cubic fourfolds via the period map, arXiv:0705.0949, (to appear in Annals of Math).

[Lo] E. Looijenga, The period map for cubic fourfolds, arXiv:0705.0951, (to appear in Invent. Math.).

$[\mathrm{N}] \quad$ V. V. Nikulin, Integer quadratic forms and some of their geometrical applications, Math. USSR - Izv. 43 (1979), 103-167.

[O] P. Orlik, Seifert manifolds, Lecture Notes in Math., Springer-Ver., Berlin - New York, vol. 291, 1972.

[R] V. A. Rohlin, Complex topological characteristics of real algebraic curves, Uspekhi Mat. Nauk 33 (1978), 77-89.

[Vi] O. Viro, Progress of the last six years in topology of real algebraic varieties, Russian Math. Surveys 41 (1986), 55-82.

[Vo] C. Voisin, Théorème de Torelli pour les cubiques de $P^{5}$, Invent. Math. 86 (1986), 577-601.

Middle East Technical University, Department of Mathematics

ANKARA 06531 TURKEY

Université de Strasbourg et IRMA (CNRS)

7 rue René Descartes 67084 Strasbourg Cedex, France 